This item was submitted to Loughborough's Research Repository by the author.

Items in Figshare are protected by copyright, with all rights reserved, unless otherwise indicated.

\title{
Numerical approximation of random periodic solutions of stochastic differential equations
}

PLEASE CITE THE PUBLISHED VERSION

https://doi.org/10.1007/s00033-017-0868-7

\section{PUBLISHER}

Springer Verlag $@$ The Author(s)

VERSION

VoR (Version of Record)

\section{PUBLISHER STATEMENT}

This work is made available according to the conditions of the Creative Commons Attribution-NonCommercialNoDerivatives 4.0 International (CC BY-NC-ND 4.0) licence. Full details of this licence are available at: https://creativecommons.org/licenses/by-nc-nd/4.0/

\section{LICENCE}

CC BY-NC-ND 4.0

\section{REPOSITORY RECORD}

Feng, Chunrong, Yu Liu, and Huaizhong Zhao. 2019. "Numerical Approximation of Random Periodic Solutions of Stochastic Differential Equations". figshare. https://hdl.handle.net/2134/26569. 


\title{
Numerical approximation of random periodic solutions of stochastic differential equations
}

\author{
Chunrong Feng, Yu Liu and Huaizhong Zhao®
}

\begin{abstract}
In this paper, we discuss the numerical approximation of random periodic solutions of stochastic differential equations (SDEs) with multiplicative noise. We prove the existence of the random periodic solution as the limit of the pull-back flow when the starting time tends to $-\infty$ along the multiple integrals of the period. As the random periodic solution is not explicitly constructible, it is useful to study the numerical approximation. We discretise the SDE using the Euler-Maruyama scheme and modified Milstein scheme. Subsequently, we obtain the existence of the random periodic solution as the limit of the pull-back of the discretised SDE. We prove that the latter is an approximated random periodic solution with an error to the exact one at the rate of $\sqrt{\Delta t}$ in the mean square sense in Euler-Maruyama method and $\Delta t$ in the Milstein method. We also obtain the weak convergence result for the approximation of the periodic measure.
\end{abstract}

Mathematics Subject Classification. 37H99, 60H10, 60H35.

Keywords. Random periodic solution, Periodic measure, Euler-Maruyama method, Modified Milstein method, Infinite horizon, Rate of convergence, Pull-back, Weak convergence.

\section{Introduction}

Periodic solution has been a central concept in the theory of dynamical systems since Poincaré's pioneering work [18-21]. As the random counterpart of periodic solution, the concept of random periodic solutions (RPS) began to be addressed recently for a $C^{1}$-cocycle in [28]. Later, the definition of random periodic solutions and their existence for semi-flows generated by non-autonomous SDEs and SPDEs with additive noise were given in $[5,6]$. Denote by $\Delta:=\left\{(t, s) \in \mathbb{R}^{2}, s \leq t\right\}$. Let $\mathbb{X}$ be a separable Banach space and $\left(\Omega, \mathcal{F}, P,\left(\theta_{t}\right)_{t \in \mathbb{R}}\right)$ be a metric dynamical system. Consider a stochastic periodic semi-flow $u: \Delta \times \Omega \times \mathbb{X} \rightarrow \mathbb{X}$ of period $\tau$, which satisfies the semi-flow relation

$$
u(t, r, \omega)=u(t, s, \omega) \circ u(s, r, \omega),
$$

and the periodic property

$$
u(t+\tau, s+\tau, \omega)=u\left(t, s, \theta_{\tau} \omega\right),
$$

for all $r \leq s \leq t$. SDEs and SPDEs with time-dependent coefficients which are periodic in time generate periodic semi-flows satisfying (1.1) and (1.2) [5-7].

Definition 1.1. [5,6] A random periodic path of period $\tau$ of the semi-flow $u: \Delta \times \Omega \times \mathbb{X} \rightarrow \mathbb{X}$ is an $\mathcal{F}$-measurable map $Y: \mathbb{R} \times \Omega \rightarrow \mathbb{X}$ such that for a.e. $\omega \in \Omega$,

$$
u(t, s, \omega) Y(s, \omega)=Y(t, \omega), Y(s+\tau, \omega)=Y\left(s, \theta_{\tau} \omega\right), \text { for any }(t, s) \in \Delta .
$$

It has been proved that random periodic solutions exist for many SDEs and SPDEs [5-7]. Recently, "equivalence" of random periodic paths and periodic measures has been proved in [8] and some results of the ergodicity of periodic measures have been obtained. Note many phenomena in the real world have 
both periodic and random nature, e.g. daily temperature, energy consumption, airline passenger volumes, $\mathrm{CO}_{2}$ concentration. The concept and its study are relevant to modelling random periodicity in the real world.

In the literature, there have been a number of recent works such as [3] on random attractors of the stochastic TJ model in climate dynamics; [2] on stochastic lattice systems; [4] on stochastic resonance; [7] for SDEs with multiplicative linear noise; and [25] on bifurcations of stochastic reaction diffusion equations. All these results are theoretical on the existence of random periodic paths.

In general, neither stationary solutions nor random periodic solutions can be constructed explicitly, so numerical approximation is another indispensable tool to study stochastic dynamics, especially to physically relevant problems. It is worth mentioning here that this is a numerical approximation of an infinite time horizon problem. There are numerous works on numerical analysis of SDEs on a finite horizon, and a number of excellent monographs $[14,17]$. However, there are only a few works on infinite horizon problems. A numerical analysis of approximation to the stationary solutions and invariant measures of SDEs through discretising the pull-back was given in $[16,22-24,26]$. Numerical approximations to stable zero solutions of SDEs were given in $[10,14]$.

In this paper, we study stochastic differential equations, which possess random periodic solutions, and approximate them by Euler-Maruyama and Milstein schemes. As far as we know, this is the first paper addressing analysis of numerical approximations of random periodic solutions. Consider the following m-dimensional SDE

$$
d X_{t}^{t_{0}}=\left[A X_{t}^{t_{0}}+f\left(t, X_{t}^{t_{0}}\right)\right] d t+g\left(t, X_{t}^{t_{0}}\right) d W_{t}
$$

with $X_{t_{0}}^{t_{0}}=\xi$, where $f: \mathbb{R} \times \mathbb{R}^{m} \rightarrow \mathbb{R}^{m}, g: \mathbb{R} \times \mathbb{R}^{m} \rightarrow \mathbb{R}^{m \times d}, A$ is a symmetric and negative-definite $m \times m$ matrix, $W_{t}$ is a two-sided Wiener process in $\mathbb{R}^{d}$ on a probability space $(\Omega, \mathcal{F}, \mathbb{P})$. The filtration is defined as follows: $\mathcal{F}_{s}^{t}=\sigma\left\{W_{u}-W_{v}: s \leq v \leq u \leq t\right\}, \mathcal{F}^{t}=\mathcal{F}_{-\infty}^{t}=\bigvee_{s \leq t} \mathcal{F}_{s}^{t}$, the random variable $\xi$ is $\mathcal{F}^{t_{0}}$-measurable. We assume that the functions $f$ and $g$ are $\tau$-periodic in time. By the variation of constant formula, the solution of (1.3) is given

$$
X_{t}^{t_{0}}(\xi)=e^{A\left(t-t_{0}\right)} \xi+e^{A t} \int_{t_{0}}^{t} e^{-A s} f\left(s, X_{s}^{t_{0}}\right) d s+e^{A t} \int_{t_{0}}^{t} e^{-A s} g\left(s, X_{s}^{t_{0}}\right) d W_{s} .
$$

Denote the standard $P$-preserving ergodic Wiener shift by $\theta: \mathbb{R} \times \Omega \rightarrow \Omega, \theta_{t}(\omega)(s):=W(t+s)-$ $W(t), t, s \in \mathbb{R}$. The solution $X$ of the non-autonomous SDE does not satisfy the cocycle property, but $u\left(t, t_{0}\right): \Omega \times R^{m} \rightarrow R^{m}$ given by $u\left(t, t_{0}\right) \xi=X_{t}^{t_{0}}(\xi)$ satisfies the semi-flow property (1.1) and periodicity (1.2). Denote by $X_{r}^{-k \tau}(\xi, \omega)$ the solution starting from time $-k \tau$. We will show that when $k \rightarrow \infty$, the pull-back $X_{r}^{-k \tau}(\xi)$ has a limit $X_{r}^{*}$ in $L^{2}(\Omega)$ and $X_{r}^{*}$ is the random periodic solution of SDE (1.3). It satisfies the infinite horizon stochastic integral equation (IHSIE)

$$
X_{r}^{*}=\int_{-\infty}^{r} e^{A(r-s)} f\left(s, X_{s}^{*}\right) d s+\int_{-\infty}^{r} e^{A(r-s)} g\left(s, X_{s}^{*}\right) d W_{s}
$$

We separate the linear term $A X$ from the nonlinear term in (1.3) to enable us to represent the random periodic solution by IHSIE $[5,7]$. This is helpful to formulate the scheme for SPDEs for which random periodic solutions were considered in [6].

Numerical analysis for random periodic solutions was not considered in previous work. The infinite horizon stochastic integral equation (IHSIE) method can deal with anticipated cases [5-7]. But it is still not clear how to numerically approximate two-sided IHSIE and anticipating random periodic solutions. The pull-back method used in this paper is a popular way to study random attractors. Here we use this to deal with stable adapted random periodic solutions of dissipative systems for the first time. The pull-back method has some advantages. First, stability can be obtained immediately. Secondly, it can deal with some dissipative equations that cannot be dealt with by the IHSIE; especially, the current IHSIE 
technique requires equations to have multiplicative linear noise or additive noise and $f$ being bounded. Thirdly in this paper, we study numerical approximations of random periodic solutions of dissipative SDEs and with the pull-back idea, a random periodic solution of the discretised system can be obtained as well.

We will first study the Euler-Maruyama numerical scheme in infinite horizon and obtain an approximating r.p.s. $\widehat{X}_{r}^{*}$. We will prove that the latter converges to the exact r.p.s. in $L^{2}(\Omega)$ at the rate of $\sqrt{\Delta t}$ when the time mesh $\sqrt{\Delta t}$ tends to zero. This result will be numerically verified. Despite its lower order of the approximation only at the rate of $\sqrt{\Delta t}$, the advantage of this scheme is its simplicity and it is relatively easy to implement in actual computations. It works well for the SDE we consider in this paper.

We also consider more advanced numerical schemes, e.g. Milstein scheme [13,14,24], for high-order convergence. We improve the rate of approximation from $\sqrt{\Delta t}$ in Euler scheme to $\Delta t$.

We will also do some numerical simulations to sample paths of the r.p.s. (Fig. 1). However, simulation of one pathwise trajectory is not a reliable way to tell whether or not it is random periodic though it looks very much like to be. Here we provide two reliable methods for this from numerical simulations. One method is to simulate $\left\{X_{t}^{*}(\omega), t \in \mathbb{R}\right\}$ and $\left\{X_{t}^{*}\left(\theta_{-\tau} \omega\right), t \in \mathbb{R}\right\}$ for the same $\omega$. These two trajectories should be repeating each other, but with a shift of one period of time. See Fig. 1 as an example. The other way is to simulate $\left\{X_{t}^{*}\left(\theta_{-t} \omega\right), t \in \mathbb{R}\right\}$, which is periodic if and only if $X_{t}^{*}(\omega)$ is random periodic. As an example, see Fig. 2. These two approaches would apply to any other stochastic differential equations should they have a random periodic solution.

It was known from the recent work [8] that the law of the random periodic solution is the periodic measure of the corresponding Markov semi-group. Thus, we will consider the convergence of transition probabilities generated by (1.3) and its numerical scheme along the integral multiples of period to the periodic measure and discretised periodic measure, respectively, and error estimate of the two periodic measures in the weak topology.

\section{Assumptions and preliminary results}

First we fix some notation. Let $p \geq 1$ and denote the $L^{p}$-norm of a random variable $\xi$ by $\|\xi\|_{p}=\left(\mathbb{E}|\xi|^{p}\right)^{1 / p}$, and the Frobenius norm of any $d_{1} \times d_{2}$ matrix $B$ by $|B|=\left(\sum_{i=1}^{d_{1}} \sum_{j=1}^{d_{2}} B_{i j}^{2}\right)^{\frac{1}{2}}$.

\subsection{Conditions for the SDE}

We assume the following conditions.

Condition (A). The eigenvalues of the symmetric matrix $A,\left\{\lambda_{j}, j=1,2, \ldots, m\right\}$, satisfy $0>\lambda_{1} \geq \lambda_{2} \geq$ $\ldots \geq \lambda_{m}$.

Condition (1). Assume there exists a constant $\tau>0$ such that for any $t \in \mathbb{R}, x \in \mathbb{R}^{m}, f(t+\tau, x)=$ $f(t, x), g(t+\tau, x)=g(t, x)$ and there exist constants $C_{0}, \beta_{1}, \beta_{2}>0$ with $\beta_{1}+\frac{\beta_{2}^{2}}{2}<\left|\lambda_{1}\right|$ such that for any $s, t \in \mathbb{R}$ and $x, y \in \mathbb{R}^{m}$,

$$
\begin{gathered}
|f(s, x)-f(t, y)| \leq C_{0}|s-t|^{1 / 2}+\beta_{1}|x-y|, \\
|g(s, x)-g(t, y)| \leq C_{0}|s-t|^{1 / 2}+\beta_{2}|x-y| .
\end{gathered}
$$

Condition (2). There exists a constant $K^{*}>0$ such that $\|\xi\|_{2} \leq K^{*}$.

From Condition (1) it follows that for any $x \in \mathbb{R}^{m}$, the linear growth condition also holds: $|f(t, x)| \leq$ $\beta_{1}|x|+C_{1},|g(t, x)| \leq \beta_{2}|x|+C_{2}$, where the constants $C_{1}, C_{2}>0$ are constants. It is easy to see that there exists a constant $\alpha$ such that $\beta_{1}+\frac{\beta_{2}^{2}}{2}<\alpha<\left|\lambda_{1}\right|$. In the following, we always assume that $\alpha$ satisfies this condition in all the following proofs. Set $\rho:=\left|\lambda_{m}\right|$. 
For the SDE case, the quantity $\rho$ is certainly finite, and for simplicity, we choose numerical schemes to treat the linear part explicitly, which simplify the proof of the pull-back convergence to the random periodic solutions for the discretised systems. However, in a case of SPDEs, this technical assumption is no longer true, but can be removed by employing exponential Euler-Maruyama method and Milstein scheme $[1,12]$. This will be studied in future work.

\subsection{Existence and uniqueness of random periodic solution}

We first consider the boundedness of the solution in $L^{2}(\Omega)$.

Lemma 2.1. Assume Conditions (A), (1) and (2). Then there exists a constant $C>0$ such that for any $k \in \mathbb{N}, r \geq-k \tau$, we have $\mathbb{E}\left|X_{r}^{-k \tau}\right|^{2} \leq C$.

Proof. First, using Itô's formula to $e^{2 \alpha r}\left|X_{r}^{-k \tau}\right|^{2}$, we have

$$
\begin{aligned}
e^{2 \alpha r}\left|X_{r}^{-k \tau}\right|^{2}= & e^{-2 \alpha k \tau}|\xi|^{2}+2 \alpha \int_{-k \tau}^{r} e^{2 \alpha s}\left|X_{s}^{-k \tau}\right|^{2} d s+2 \int_{-k \tau}^{r} e^{2 \alpha s}\left(X_{s}^{-k \tau}\right)^{T} A X_{s}^{-k \tau} d s \\
& +2 \int_{-k \tau}^{r} e^{2 \alpha s}\left(X_{s}^{-k \tau}\right)^{T} f\left(s, X_{s}^{-k \tau}\right) d s+\int_{-k \tau}^{r} e^{2 \alpha s}\left|g\left(s, X_{s}^{-k \tau}\right)\right|^{2} d s \\
& +2 \int_{-k \tau}^{r} e^{2 \alpha s}\left(X_{s}^{-k \tau}\right)^{T} g\left(s, X_{s}^{-k \tau}\right) d W_{s} .
\end{aligned}
$$

Firstly, note the sum of the second and third terms of the right-hand side is non-positive as the matrix $\alpha I+A$ is non-positive definite. Take the expectation of both sides of (2.1), apply the above inequality and use linear growth conditions to obtain

$$
\begin{aligned}
e^{2 \alpha r} \mathbb{E}\left|X_{r}^{-k \tau}\right|^{2} \leq & e^{-2 \alpha k \tau}\|\xi\|_{2}^{2}+\left(2 \beta_{1}+\beta_{2}^{2}\right) \int_{-k \tau}^{r} e^{2 \alpha s} \mathbb{E}\left|X_{s}^{-k \tau}\right|^{2} d s \\
& +2\left(C_{1}+\beta_{2} C_{2}\right) \int_{-k \tau}^{r} e^{2 \alpha s} \mathbb{E}\left|X_{s}^{-k \tau}\right| d s+(2 \alpha)^{-1} C_{2}^{2}\left(e^{2 \alpha r}-e^{-2 \alpha k \tau}\right) .
\end{aligned}
$$

Also, there exits $\varepsilon>0$, such that $\left(\beta_{1}+\frac{\beta_{2}^{2}}{2}\right)(1+\varepsilon)<\alpha<\left|\lambda_{1}\right|$. By Young's inequality

$$
2\left(C_{1}+\beta_{2} C_{2}\right)\left|X_{s}^{-k \tau}\right| \leq \frac{\left(C_{1}+\beta_{2} C_{2}\right)^{2}}{\varepsilon\left(2 \beta_{1}+\beta_{2}^{2}\right)}+\varepsilon\left(2 \beta_{1}+\beta_{2}^{2}\right)\left|X_{s}^{-k \tau}\right|^{2} .
$$

Then we have

$$
e^{2 \alpha r} \mathbb{E}\left|X_{r}^{-k \tau}\right|^{2} \leq K_{1}+K_{2} e^{2 \alpha r}+K_{3} \int_{-k \tau}^{r} e^{2 \alpha s}\left\|X_{s}^{-k \tau}\right\|_{2}^{2} d s
$$

where

$$
\begin{aligned}
& K_{1}=e^{-2 \alpha k \tau}\|\xi\|_{2}^{2}-\left(\frac{C_{2}^{2}}{2 \alpha}+\frac{\left(C_{1}+\beta_{2} C_{2}\right)^{2}}{2 \alpha \varepsilon\left(2 \beta_{1}+\beta_{2}^{2}\right)}\right) e^{-2 \alpha k \tau} \\
& K_{2}=\frac{C_{2}^{2}}{2 \alpha}+\frac{\left(C_{1}+\beta_{2} C_{2}\right)^{2}}{2 \alpha \varepsilon\left(2 \beta_{1}+\beta_{2}^{2}\right)}, K_{3}=\left(2 \beta_{1}+\beta_{2}^{2}\right)(1+\varepsilon)<2 \alpha .
\end{aligned}
$$


Now applying Gronwall's inequality, we have

$$
\begin{aligned}
& e^{2 \alpha r} \mathbb{E}\left|X_{r}^{-k \tau}\right|^{2} \leq K_{1}+K_{2} e^{2 \alpha r}+\int_{-k \tau}^{r}\left(K_{1}+K_{2} e^{2 \alpha s}\right) K_{3} e^{\int^{r} K_{3} d r} d s \\
& \leq\left(K_{1} e^{2 \alpha k \tau}+K_{2}\right) e^{2 \alpha r}+\frac{K_{2} K_{3}}{2 \alpha-K_{3}} e^{2 \alpha r} .
\end{aligned}
$$

Here we notice that $K_{1} e^{2 \alpha k \tau}+K_{2}=\|\xi\|_{2}^{2}$. Therefore, by Condition (2)

$$
\mathbb{E}\left|X_{r}^{-k \tau}\right|^{2} \leq\|\xi\|_{2}^{2}+\frac{2 \alpha K_{2}}{2 \alpha-K_{3}} \leq K^{*}+\frac{2 \alpha K_{2}}{2 \alpha-K_{3}}
$$

In the next lemma, we will also obtain a bound on the norm $\left\|X_{t_{1}}^{-k \tau}-X_{t_{2}}^{-k \tau}\right\|_{2}$ for any fixed time $t_{1}, t_{2}$. This will be essential for us to estimate the error of the numerical approximation in Sect. 4.

Lemma 2.2. Assume Conditions (A), (1) and (2). Then there exist constants $C_{3}>0, C_{4}>0$, such that for any positive $k \in \mathbb{N}$ and any $t_{1}, t_{2} \geq 0, t_{1} \geq t_{2}$, the solution of (1.3) satisfies $\left\|X_{t_{1}}^{-k \tau}-X_{t_{2}}^{-k \tau}\right\|_{2} \leq$ $C_{3}\left(t_{1}-t_{2}\right)+C_{4} \sqrt{t_{1}-t_{2}}$.

Proof. From (1.4), we see that

$$
\begin{aligned}
\left\|X_{t_{1}}^{-k \tau}-X_{t_{2}}^{-k \tau}\right\|_{2} \leq & e^{2 A k \tau}\|\xi\|_{2}\left|e^{A t_{1}}-e^{A t_{2}}\right| \\
& +\left\|e^{A t_{1}} \int_{-k \tau}^{t_{1}} e^{-A s} f\left(s, X_{s}^{-k \tau}\right) d s-e^{A t_{2}} \int_{-k \tau}^{t_{2}} e^{-A s} f\left(s, X_{s}^{-k \tau}\right) d s\right\|_{2} \\
& +\left\|e^{A t_{1}} \int_{-k \tau}^{t_{1}} e^{-A s} g\left(s, X_{s}^{-k \tau}\right) d W_{s}-e^{A t_{2}} \int_{-k \tau}^{t_{2}} e^{-A s} g\left(s, X_{s}^{-k \tau}\right) d W_{s}\right\|_{2} .
\end{aligned}
$$

We evaluate each term on the right-hand side of (2.2). First, we consider the first term. By Lemma 1 in [26], $\left|e^{A t_{1}}-e^{A t_{2}}\right| \leq|A|\left(t_{1}-t_{2}\right)$. Now we estimate the third term with the Minkowski inequality, Itô's isometry and the linear growth property:

$$
\begin{aligned}
& \left\|e^{A t_{1}} \int_{-k \tau}^{t_{1}} e^{-A s} g\left(s, X_{s}^{-k \tau}\right) d W_{s}-e^{A t_{2}} \int_{-k \tau}^{t_{2}} e^{-A s} g\left(s, X_{s}^{-k \tau}\right) d W_{s}\right\|_{2} \\
& \leq\left\|\int_{-k \tau}^{t_{2}}\left(e^{A t_{1}}-e^{A t_{2}}\right) e^{-A s} g\left(s, X_{s}^{-k \tau}\right) d W_{s}\right\|_{2}+\left\|\int_{t_{2}}^{t_{1}} e^{-A\left(s-t_{1}\right)} g\left(s, X_{s}^{-k \tau}\right) d W_{s}\right\|_{2} \\
& \leq \sqrt{\int_{-k \tau}^{t_{2}}\left|\left(e^{A t_{1}}-e^{A t_{2}}\right) e^{-A s}\right|^{2} \mathbb{E}\left[\beta_{2}\left(\left|X_{s}^{-k \tau}\right|\right)+C_{2}\right]^{2} d s} \\
& \quad+\sqrt{\int_{t_{2}}^{t_{1}} \mid e^{-\left.A\left(s-t_{1}\right)\right|^{2} \mathbb{E}\left[\beta_{2}\left(\left|X_{s}^{-k \tau}\right|\right)+C_{2}\right]^{2} d s}} \\
& \leq \sqrt{\int_{-k \tau}^{t_{2}}\left|\left(e^{A t_{1}}-e^{A t_{2}}\right) e^{-A s}\right|^{2}\left(2 \beta_{2}^{2} \mathbb{E}\left|X_{s}^{-k \tau}\right|^{2}+2 C_{2}^{2}\right) d s}
\end{aligned}
$$




$$
\begin{aligned}
& +\sqrt{\int_{t_{2}}^{t_{1}}\left|e^{-A\left(s-t_{1}\right)}\right|^{2}\left(2 \beta_{2}^{2} \mathbb{E}\left|X_{s}^{-k \tau}\right|^{2}+2 C_{2}^{2}\right) d s} \\
& \leq K_{4} \sqrt{\int_{-k \tau}^{t_{2}}\left|\left(e^{A t_{1}}-e^{A t_{2}}\right) e^{-A s}\right|^{2} d s+K_{4} \sqrt{\int_{t_{2}}^{t_{1}}\left|e^{-A\left(s-t_{1}\right)}\right|^{2} d s .}} \\
& \leq K_{4} \sqrt{\frac{\operatorname{Tr}(-A)}{2}\left(t_{1}-t_{2}\right)+K_{4} \sqrt{t_{1}-t_{2}}} .
\end{aligned}
$$

Here we take some constant $K_{4}$ because $\mathbb{E}\left|X_{s}^{-k \tau}\right|^{2}$ is bounded above according to Lemma 2.1.

Lastly, we consider the second term of (2.2) with Minkowski inequality

$$
\begin{aligned}
\left\|e^{A t_{1}} \int_{-k \tau}^{t_{1}} e^{-A s} f\left(s, X_{s}^{-k \tau}\right) d s-e^{A t_{2}} \int_{-k \tau}^{t_{2}} e^{-A s} f\left(s, X_{s}^{-k \tau}\right) d s\right\|_{2} \\
\leq\left\|\int_{-k \tau}^{t_{2}}\left(e^{A t_{1}}-e^{A t_{2}}\right) e^{-A s} f\left(s, X_{s}^{-k \tau}\right) d s\right\|_{2}+\left\|\int_{t_{2}}^{t_{1}} e^{-A\left(s-t_{1}\right)} f\left(s, X_{s}^{-k \tau}\right) d s\right\|_{2} \\
\leq \int_{-k \tau}^{t_{2}}\left\|\left(e^{A t_{1}}-e^{A t_{2}}\right) e^{-A s} f\left(s, X_{s}^{-k \tau}\right)\right\|_{2} d s+\int_{t_{2}}^{t_{1}}\left\|e^{-A\left(s-t_{1}\right)} f\left(s, X_{s}^{-k \tau}\right)\right\|_{2} d s \\
\leq \int_{-k \tau}^{t_{2}}\left|\left(e^{A t_{1}}-e^{A t_{2}}\right) e^{-A s}\right|\left\|f\left(s, X_{s}^{-k \tau}\right)\right\|_{2} d s+\int_{t_{2}}^{t_{1}}\left|e^{-A\left(s-t_{1}\right)}\right|\left\|f\left(s, X_{s}^{-k \tau}\right)\right\|_{2} d s \\
\leq K_{5}\left(\int_{-k \tau}^{t_{2}}\left|\left(e^{A t_{1}}-e^{A t_{2}}\right) e^{-A s}\right| d s+\int_{t_{2}}^{t_{1}}\left|e^{-A\left(s-t_{1}\right)}\right| d s\right) \\
\leq 2 K_{5}\left(t_{1}-t_{2}\right),
\end{aligned}
$$

for a constant $K_{5}>0$. Combining the above estimates we obtain the lemma with the constants $C_{3}, C_{4}$ being independent of $k$ and $t_{1}, t_{2}$.

Now we continue to consider the difference of the solutions under various initial values. For simplicity, we here study two different initial values $\xi$ and $\eta$.

Lemma 2.3. Denote by $X_{r}^{-k \tau}$ and $Y_{r}^{-k \tau}$ two solutions of (1.3) with different initial values $\xi$ and $\eta$, respectively. Assume Conditions (A), (1) and Condition (2) for both initial values. Then $\left\|X_{r}^{-k \tau}-Y_{r}^{-k \tau}\right\|_{2} \leq$ $e^{\left(\beta_{1}+\frac{\beta_{2}^{2}}{2}-\alpha\right)(r+k \tau)}\|\xi-\eta\|_{2}$.

Proof. According to (1.4) we have

$$
\begin{aligned}
X_{r}^{-k \tau}-Y_{r}^{-k \tau}= & e^{A(r+k \tau)}(\xi-\eta)+e^{A r} \int_{-k \tau}^{r} e^{-A s}\left(f\left(s, X_{s}^{-k \tau}\right)-f\left(s, Y_{s}^{-k \tau}\right)\right) d s \\
& +e^{A r} \int_{-k \tau}^{r} e^{-A s}\left(g\left(s, X_{s}^{-k \tau}\right)-g\left(s, Y_{s}^{-k \tau}\right)\right) d W_{s} .
\end{aligned}
$$


For simplicity, denote $\zeta_{r}^{-k \tau}=X_{r}^{-k \tau}-Y_{r}^{-k \tau}$. Then according to the method used in Lemma 2.1, and the global Lipschitz condition, we have

$$
\begin{aligned}
e^{2 \alpha r}\left\|\zeta_{r}^{-k \tau}\right\|_{2}^{2} \leq & e^{-2 \alpha k \tau}\|\xi-\eta\|_{2}^{2}+2 \int_{-k \tau}^{r} e^{2 \alpha s} \mathbb{E}\left[( \zeta _ { s } ^ { - k \tau } ) ^ { T } \left(f\left(s, X_{s}^{-k \tau}\right)\right.\right. \\
& \left.\left.-f\left(s, Y_{s}^{-k \tau}\right)\right)\right] d s+\int_{-k \tau}^{r} e^{2 \alpha s} \mathbb{E}\left|g\left(s, X_{s}^{-k \tau}\right)-g\left(s, Y_{s}^{-k \tau}\right)\right|^{2} d s . \\
\leq & e^{-2 \alpha k \tau}\|\xi-\eta\|_{2}^{2}+\left(2 \beta_{1}+\beta_{2}^{2}\right) \int_{-k \tau}^{r} e^{2 \alpha s}\left\|\zeta_{s}^{-k \tau}\right\|_{2}^{2} d s .
\end{aligned}
$$

Then the result follows from the Gronwall inequality.

Now we can prove the following theorem.

Theorem 2.4. Assume Conditions (A), (1). Then there exists a unique random periodic solution $X^{*}(r, \cdot) \in$ $L^{2}(\Omega), r \geq 0$ such that for any initial value $\xi$ satisfying Condition (2), the solution of (1.3) satisfies $\lim _{k \rightarrow \infty}\left\|X_{r}^{-k \tau}(\xi)-X^{*}(r)\right\|_{2}=0$.

Proof. Condition (2) implies that the initial value $\xi$ belongs to $L^{2}(\Omega)$. According to Lemma $2.1, X_{r}^{-k \tau}(\cdot)$ maps $L^{2}(\Omega)$ to itself. Now we use the semi-flow property to get that for any $r, k, p \geq 0, X_{r}^{-k \tau-p \tau}(\xi)=$ $X_{r}^{-k \tau}(\omega) \circ X_{-k \tau}^{-(k+p) \tau}(\omega, \xi)$. Thus, we can apply Lemma 2.3 to have for any $\varepsilon>0$ there exists $k^{*}>0$ such that for any $k \geq k^{*},\left\|X_{r}^{-k \tau}(\xi)-X_{r}^{-(k+p) \tau}(\xi)\right\|_{2}<\varepsilon$. This means that there exists $N>0$ such that for any $l, m \geq N$, we have $\left\|X_{r}^{-l \tau}(\xi)-X_{r}^{-m \tau}(\xi)\right\|_{2}<\varepsilon$, i.e. $\left\{X_{r}^{-k \tau}(\xi)\right\}_{k \in \mathbb{N}}$ is a Cauchy sequence, so converges to some $X^{*}(r, \omega)$ in $L^{2}(\Omega)$, when $k \rightarrow \infty$.

Set $u(t, r)(\xi)=X_{t}^{r}(\xi)$, then $u(t, r): \Omega \times \mathbb{R}^{m} \rightarrow \mathbb{R}^{m}$ defines a semi-flow of homeomorphism (Kunita [15]). By the continuity of $X_{t}^{r}(\omega): L^{2}\left(\Omega, \mathbb{R}^{m}\right) \rightarrow L^{2}\left(\Omega, \mathbb{R}^{m}\right), t \geq r$, then $u(t, r, \omega)\left(X_{r}^{-k \tau}(\xi, \omega)\right) \frac{k \rightarrow \infty}{L^{2}(\Omega)}$ $u(t, r, \omega) \circ\left(X^{*}(r, \omega)\right)$. But

$$
u(t, r, \omega)\left(X_{r}^{-k \tau}(\xi, \omega)\right)=X_{t}^{-k \tau}(\xi, \omega) \underset{L^{2}(\Omega)}{\stackrel{k \rightarrow \infty}{\longrightarrow}} X^{*}(t, \omega) .
$$

So $u(t, r, \omega)\left(X^{*}(r, \omega)\right)=X^{*}(t, \omega), \mathbb{P}-$ a.s.

Taking some other initial value $\eta$ satisfying Condition (2), we have

$$
\left\|X_{r}^{*}-X_{r}^{-k \tau}(\eta)\right\|_{2} \leq\left\|X_{r}^{*}-X_{r}^{-k \tau}(\xi)\right\|_{2}+\left\|X_{r}^{-k \tau}(\xi)-X_{r}^{-k \tau}(\eta)\right\|_{2} .
$$

Applying Lemma 2.3 again, we can make the right-hand side small enough when $k \rightarrow \infty$. Therefore, the convergence is independent of the initial value.

Now we need to prove the random periodicity of the $X^{*}(r, \omega)$. Note by the continuity of $f$ and $g$,

$$
X_{r+\tau}^{-(k-1) \tau}(\xi)=e^{A(r+k \tau)} \xi+\int_{-k \tau}^{r} e^{A(r-s)}\left[f\left(s, X_{s+\tau}^{-(k-1) \tau}(\xi)\right) d s+g\left(s, X_{s+\tau}^{-(k-1) \tau}(\xi)\right) d \widetilde{W}_{s}\right],
$$

where $\widetilde{W}_{s}:=\left(\theta_{\tau} \omega\right)(s)=W_{s+\tau}-W_{\tau}$. On the other hand,

$$
\theta_{\tau} X_{-k \tau}^{r}(\xi)=e^{A(r+k \tau)} \theta_{\tau} \xi+\int_{-k \tau}^{r} e^{A(r-s)}\left[f\left(s, \theta_{\tau} X_{s}^{-k \tau}\right) d s+g\left(s, \theta_{\tau} X_{s}^{-k \tau}\right) d \widetilde{W}_{s}\right] .
$$


By pathwise uniqueness of the solution of (1.3), we have

$$
X_{r}^{-k \tau}\left(\theta_{\tau} \omega, \xi\left(\theta_{\tau} \omega\right)\right)=\theta_{\tau} X_{r}^{-k \tau}(\xi)=X_{r+\tau}^{-(k-1) \tau}(\omega, \xi(\omega)) .
$$

From the proof of convergence we have

$$
X_{r+\tau}^{-(k-1) \tau}(\omega, \xi) \underset{L^{2}(\Omega)}{\stackrel{k \rightarrow \infty}{\longrightarrow}} X^{*}(r+\tau, \omega), \quad X_{r}^{-k \tau}\left(\theta_{\tau} \omega, \xi\left(\theta_{\tau} \omega\right)\right) \underset{L^{2}(\Omega)}{\stackrel{k \rightarrow \infty}{\longrightarrow}} X^{*}\left(r, \theta_{\tau} \omega\right) .
$$

Therefore, $X^{*}(r+\tau, \omega)=X^{*}\left(r, \theta_{\tau} \omega\right), \mathbb{P}-$ a.s.

\section{Numerical approximation for random periodic solution}

\subsection{Euler-Maruyama scheme}

In this section, we will introduce the basic Euler-Maruyama method to approximate the solution on infinite horizon. Take $\Delta t=\tau / n$, which will be taken to be sufficiently small such that $\Delta t \leq \frac{1}{\rho}$, for some $n \in \mathbb{N}$, in the remaining part of the paper. Let $N=k n$. The time domain from time $-k \tau$ to time 0 is divided into $N$ intervals of length $\Delta t$ such that $N \Delta t=k \tau$. The scheme starts from an $\mathcal{F}^{-k \tau}$-measurable random variable $\xi$ at a time $-k \tau$. At each of the points $i \Delta t$ we set the value $\widehat{X}_{-k \tau+i \Delta t}^{-k \tau}$ with the iteration formula

$$
\begin{aligned}
\widehat{X}_{-k \tau+(i+1) \Delta t}^{-k \tau}= & \widehat{X}_{-k \tau+i \Delta t}^{-k \tau}+A \widehat{X}_{-k \tau+i \Delta t}^{-k \tau} \Delta t+f\left(i \Delta t, \widehat{X}_{-k \tau+i \Delta t}^{-k \tau}\right) \Delta t \\
& +g\left(i \Delta t, \widehat{X}_{-k \tau+i \Delta t}^{-k \tau}\right)\left(W_{-k \tau+(i+1) \Delta t}-W_{-k \tau+i \Delta t}\right),
\end{aligned}
$$

where $i=0,1,2, \ldots$, and $\widehat{X}_{-k \tau+0 \Delta t}^{-k \tau}=\xi$.

It is easy to see that for any $M \geq 0$,

$$
\begin{aligned}
\widehat{X}_{-k \tau+M \Delta t}^{-k \tau}= & (I+A \Delta t)^{M} \xi+\Delta t \sum_{i=0}^{M-1}(I+A \Delta t)^{M-i-1} f\left(i \Delta t, \widehat{X}_{-k \tau+i \Delta t}^{-k \tau}\right) \\
& +\sum_{i=0}^{M-1}(I+A \Delta t)^{M-i-1} g\left(i \Delta t, \widehat{X}_{-k \tau+i \Delta t}^{-k \tau}\right)\left(W_{-k \tau+(i+1) \Delta t}-W_{-k \tau+i \Delta t}\right) .
\end{aligned}
$$

Moreover, we can set up a discrete semi-flow given by $\hat{u}_{i, j}(\xi)=\hat{X}_{i \Delta t}^{j \Delta t}(\xi), i \geq j, i, j \in\{-k n,-k n+$ $1, \cdots\}, \hat{\theta}=\theta_{\Delta t}, \hat{\theta}^{n}=\hat{\theta} \hat{\theta} \cdots \hat{\theta}$. Then it is easy to see that $u$ satisfies the semi-flow property $\hat{u}_{i, j}(\omega) \circ$ $\hat{u}_{j, l}(\omega)=\hat{u}_{i, l}(\omega)$, for $i \geq j \geq l$, and the periodic property $\hat{u}_{i+n, j+n}(\omega)=\hat{u}_{i, j}\left(\hat{\theta}^{n} \omega\right)$ for $i \geq j$.

In order to prove the convergence of the discretised semi-flow to a random periodic solution, we first derive some similar estimates as in Lemmas 2.1 and 2.3. Then a discrete analogue of Theorem 2.4 will give us the result.

Lemma 3.1. Assume Conditions (A), (1) and (2). Then there exists a constant $\widehat{C}>0$ such that for any natural numbers $k \geq 0, M \geq 0$, and sufficiently small $\Delta t$, the numerical solution $\widehat{X}_{-k \tau+M \Delta t}^{-k \tau}$ defined by (3.2) satisfies $\mathbb{E}\left|\widehat{X}_{-k \tau+M \Delta t}^{-k \tau}\right|^{2} \leq \widehat{C}$.

Proof. We still choose $\alpha$ such that $\beta_{1}+\frac{\beta^{2}}{2}<\alpha<\left|\lambda_{1}\right|$. Then for any $M \geq 0$,

$$
\begin{aligned}
& (1-\alpha \Delta t)^{-2 M}\left|\widehat{X}_{-k \tau+M \Delta t}^{-k \tau}\right|^{2} \\
& \quad=|\xi|^{2}+\sum_{i=0}^{M-1}(1-\alpha \Delta t)^{-2 i}\left(\frac{\left|\widehat{X}_{-k \tau+(i+1) \Delta t}^{-k \tau}\right|^{2}}{(1-\alpha \Delta t)^{2}}-\left|\widehat{X}_{-k \tau+i \Delta t}^{-k \tau}\right|^{2}\right) .
\end{aligned}
$$


This is not hard to verify by expanding the sum and noting cancellations. Notice that

$$
\begin{aligned}
& \frac{\left|\widehat{X}_{-k \tau+(i+1) \Delta t}^{-k \tau}\right|^{2}}{(1-\alpha \Delta t)^{2}}-\left|\widehat{X}_{-k \tau+i \Delta t}^{-k \tau}\right|^{2} \\
& =\left(\left(\widehat{X}_{-k \tau+i \Delta t}^{-k \tau}\right)\left(\frac{I+A \Delta t}{1-\alpha \Delta t}-I\right)+\frac{\Delta t}{1-\alpha \Delta t} f\left(i \Delta t, \widehat{X}_{-k \tau+i \Delta t}^{-k \tau}\right)^{T}\right. \\
& \left.\quad+\frac{\left(W_{-k \tau+(i+1) \Delta t}-W_{-k \tau+i \Delta t}\right)^{T} g\left(i \Delta t, \widehat{X}_{-k \tau+i \Delta t}^{-k \tau}\right)^{T}}{1-\alpha \Delta t}\right) \\
& \quad \times\left(\left(\frac{I+A \Delta t}{1-\alpha \Delta t}+I\right) \widehat{X}_{-k \tau+i \Delta t}^{-k \tau}+\frac{\Delta t}{1-\alpha \Delta t} f\left(i \Delta t, \widehat{X}_{-k \tau+i \Delta t}^{-k \tau}\right)\right. \\
& \left.\quad+\frac{g\left(i \Delta t, \widehat{X}_{-k \tau+i \Delta t}^{-k \tau}\right)\left(W_{-k \tau+(i+1) \Delta t}-W_{-k \tau+i \Delta t}\right)}{1-\alpha \Delta t}\right) .
\end{aligned}
$$

Note $\left(\frac{I+A \Delta t}{1-\alpha \Delta t}-I\right)\left(\frac{I+A \Delta t}{1-\alpha \Delta t}+I\right)$ is non-positive definite, where $\Delta t$ satisfies $0<\Delta t \leq \frac{1}{\rho}$ as defined before, and for each $i, f\left(i \Delta t, \widehat{X}_{-k \tau+i \Delta t}^{-k \tau}\right)$ and $g\left(i \Delta t, \widehat{X}_{-k \tau+i \Delta t}^{-k \tau}\right)$ are both independent of $\left(W_{-k \tau+(i+1) \Delta t}-W_{-k \tau+i \Delta t}\right)$. Take expectation on both sides of (3.3), consider (3.4), apply the linear growth property and Young's inequality to have

$$
\begin{aligned}
&(1-\alpha \Delta t)^{-2 M} \mathbb{E}\left|\widehat{X}_{-k \tau+M \Delta t}^{-k \tau}\right|^{2} \\
& \leq\|\xi\|_{2}^{2}+\sum_{i=0}^{M-1}(1-\alpha \Delta t)^{-2 i}\left(\frac{\Delta t}{1-\alpha \Delta t}\right)^{2} \mathbb{E}\left|f\left(i \Delta t, \widehat{X}_{-k \tau+i \Delta t}^{-k \tau}\right)\right|^{2} \\
&+\sum_{i=0}^{M-1}(1-\alpha \Delta t)^{-2 i} \frac{\Delta t}{(1-\alpha \Delta t)^{2}} \mathbb{E}\left|g\left(i \Delta t, \widehat{X}_{-k \tau+i \Delta t}^{-k \tau}\right)\right|^{2} \\
&+\sum_{i=0}^{M-1}(1-\alpha \Delta t)^{-2 i} \frac{2 \Delta t}{(1-\alpha \Delta t)^{2}} \mathbb{E}\left[\left(\widehat{X}_{-k \tau+i \Delta t}^{-k \tau}\right)^{T}(I+A \Delta t) f\left(i \Delta t, \widehat{X}_{-k \tau+i \Delta t}^{-k \tau}\right)\right] \\
& \leq \widehat{K}_{1}+(1-\alpha \Delta t)^{-2 M} \widehat{K}_{2}+\widehat{K}_{3} \sum_{i=0}^{M-1}(1-\alpha \Delta t)^{-2 i} \mathbb{E}\left|\widehat{X}_{-k \tau+i \Delta t}^{-k \tau}\right|^{2},
\end{aligned}
$$

where

$$
\begin{aligned}
& \widehat{K}_{1}=\|\xi\|_{2}^{2}, \widehat{K}_{3}=\frac{\Delta t}{(1-\alpha \Delta t)^{2}}(1+\widehat{\varepsilon})\left(2 \beta_{1}+\beta_{2}^{2}+\Delta t\left(\beta_{1}^{2}+2 \beta_{1}|A|\right)\right), \\
& \widehat{K}_{2}=\frac{C_{1}^{2}(\Delta t)^{2}+C_{2}^{2} \Delta t}{2 \alpha \Delta t-\alpha^{2}(\Delta t)^{2}}+\frac{\Delta t}{2 \alpha \Delta t-\alpha^{2}(\Delta t)^{2}} \frac{\left(C_{1}+\beta_{2} C_{2}+\Delta t C_{1}\left(\beta_{1}+|A|\right)\right)^{2}}{\widehat{\varepsilon}\left(2 \beta_{1}+\beta_{2}^{2}+\Delta t\left(\beta_{1}^{2}+2 \beta_{1}|A|\right)\right)} .
\end{aligned}
$$

Here $\Delta t$ and $\widehat{\varepsilon}$ need to be chosen small enough such that

$$
(1+\widehat{\varepsilon})\left(2 \beta_{1}+\beta_{2}^{2}+\Delta t\left(\beta_{1}^{2}+2 \beta_{1}|A|\right)\right)+\alpha^{2} \Delta t<2 \alpha .
$$


This guarantees that $(1-\alpha \Delta t)^{2}\left(1+\widehat{K}_{3}\right)<1$. By the discrete Gronwall inequality,

$$
\begin{aligned}
& (1-\alpha \Delta t)^{-2 M} \mathbb{E}\left|\widehat{X}_{-k \tau+M \Delta t}^{-k \tau}\right|^{2} \\
& \quad \leq \widehat{K}_{1}+\widehat{K}_{2}(1-\alpha \Delta t)^{-2 M}+\sum_{i=0}^{M-1}\left(\widehat{K}_{1}+\widehat{K}_{2}(1-\alpha \Delta t)^{-2 i}\right) \widehat{K}_{3}\left(1+\widehat{K}_{3}\right)^{M-i-1} .
\end{aligned}
$$

It turns out that

$$
\begin{aligned}
\mathbb{E}\left|\widehat{X}_{-k \tau+M \Delta t}^{-k \tau}\right|^{2} \leq & \widehat{K}_{2}+\widehat{K}_{1}\left(\left(1+\widehat{K}_{3}\right)(1-\alpha \Delta t)^{2}\right)^{M} \\
& +\frac{\widehat{K}_{2} \widehat{K}_{3}(1-\alpha \Delta t)^{2}\left(1-\left(\left(1+\widehat{K}_{3}\right)(1-\alpha \Delta t)^{2}\right)^{M}\right)}{1-\left(1+\widehat{K}_{3}\right)(1-\alpha \Delta t)^{2}} \leq \widehat{C} .
\end{aligned}
$$

Note the choice of the constant $\widehat{C}$ is independent of $k$ and the lemma holds for sufficiently small time step $\Delta t$ and constant $\widehat{\varepsilon}$.

The following lemma is a discrete analogue of Lemma 2.3.

Lemma 3.2. Denote by $\widehat{X}_{-k \tau+M \Delta t}^{-k \tau}$ and $\widehat{Y}_{-k \tau+M \Delta t}^{-k \tau}$ solutions of the Euler scheme with initial values $\xi$ and $\eta$, respectively. Assume Conditions (A), (1) and Condition (2) for both initial values. Let $\Delta t=\tau / n$, $n \in \mathbb{Z}^{+}$, be sufficiently small such that $0<\Delta t \leq \frac{1}{\rho}$. Then for any $\varepsilon>0$, there exists an integer $M^{*}>0$ such that for any $M \geq M^{*}$, we have $\left\|\widehat{X}_{-k \tau+M \Delta t}^{-k \tau}-\widehat{Y}_{-k \tau+M \Delta t}^{-k \tau}\right\|_{2}<\varepsilon$.

Proof. According to scheme (3.2) we have

$$
\begin{aligned}
\widehat{X}_{-k \tau+M \Delta t}^{-k \tau}-\widehat{Y}_{-k \tau+M \Delta t}^{-k \tau}= & (I+A \Delta t)^{M}(\xi-\eta)+\Delta t \sum_{i=0}^{M-1}(I+A \Delta t)^{M-i-1} \widehat{F}_{i} \\
& +\sum_{i=0}^{M-1}(I+A \Delta t)^{M-i-1} \widehat{G}_{i}\left(W_{-k \tau+(i+1) \Delta t}-W_{-k \tau+i \Delta t}\right) .
\end{aligned}
$$

Here $\widehat{F}_{i}=f\left(i \Delta t, \widehat{X}_{-k \tau+i \Delta t}^{-k \tau}\right)-f\left(i \Delta t, \widehat{Y}_{-k \tau+i \Delta t}^{-k \tau}\right), \widehat{G}_{i}=g\left(i \Delta t, \widehat{X}_{-k \tau+i \Delta t}^{-k \tau}\right)-g(i \Delta t$, $\left.\widehat{Y}_{-k \tau+i \Delta t}^{-k \tau}\right)$. Denote $\widehat{\zeta}_{i}=\widehat{X}_{-k \tau+i \Delta t}^{-k \tau}-\widehat{Y}_{-k \tau+i \Delta t}^{-k \tau}$. Then by Condition (1), we have $\left|\widehat{F}_{i}\right| \leq \beta_{1}\left|\widehat{\zeta}_{i}\right|$ and $\left|\widehat{G}_{i}\right| \leq$ $\beta_{2}\left|\widehat{\zeta}_{i}\right|$. According to the method used in Lemma 3.1, we get the following result similar to inequality $(3.5)$

$$
\begin{aligned}
(1-\alpha \Delta t)^{-2 M} \mathbb{E}\left|\widehat{\zeta}_{M}\right|^{2} \leq & \|\xi-\eta\|_{2}^{2}+\sum_{i=0}^{M-1}(1-\alpha \Delta t)^{-2 i}\left(\frac{\Delta t}{1-\alpha \Delta t}\right)^{2} \mathbb{E}\left|\widehat{F}_{i}\right|^{2} \\
& +\sum_{i=0}^{M-1}(1-\alpha \Delta t)^{-2 i} \frac{\Delta t}{(1-\alpha \Delta t)^{2}} \mathbb{E}\left|\widehat{G}_{i}\right|^{2} \\
& +\sum_{i=0}^{M-1}(1-\alpha \Delta t)^{-2 i} \frac{2 \Delta t}{(1-\alpha \Delta t)^{2}} \mathbb{E}\left[\left(\widehat{\zeta}_{i}\right)^{T}(I+A \Delta t) \widehat{F}_{i}\right] \\
\leq & \|\xi-\eta\|_{2}^{2}+\widehat{K}_{4} \sum_{i=0}^{M-1}(1-\alpha \Delta t)^{-2 i} \mathbb{E}\left|\widehat{\zeta}_{i}\right|^{2},
\end{aligned}
$$


where $\widehat{K}_{4}=\frac{\Delta t}{(1-\alpha \Delta t)^{2}}\left(2 \beta_{1}+\beta_{2}^{2}+\Delta t\left(\beta_{1}^{2}+2 \beta_{1}|A|\right)\right)$. We choose $\Delta t$ small enough such that $2 \beta_{1}+\beta_{2}^{2}+$ $\Delta t\left(\beta_{1}^{2}+2 \beta_{1}|A|\right)+\alpha^{2} \Delta t<2 \alpha$. Then, we have $(1-\alpha \Delta t)^{2}\left(1+\widehat{K}_{4}\right)<1$. Again the discrete Gronwall inequality implies

$$
(1-\alpha \Delta t)^{-2 M} \mathbb{E}\left|\widehat{\zeta}_{M}\right|^{2} \leq\|\xi-\eta\|_{2}^{2} \prod_{i=0}^{M-1}\left(1+\widehat{K}_{4}\right)=\|\xi-\eta\|_{2}^{2}\left(1+\widehat{K}_{4}\right)^{M} .
$$

Finally, $\mathbb{E}\left|\widehat{\zeta}_{M}\right|^{2} \leq\|\xi-\eta\|_{2}^{2}\left((1-\alpha \Delta t)^{2}\left(1+\widehat{K}_{4}\right)\right)^{M}<\varepsilon$ with sufficiently large $M$.

In the numerical scheme we consider the process as two parts, $[-k \tau, 0)$ and $[0, r]$. Define

$$
\widehat{X}_{r}^{-k \tau}:=\widehat{X}(r, 0, \omega) \circ \widehat{X}_{0}^{-k \tau},
$$

where $\widehat{X}(r, 0, \omega), r \geq 0$, is finite time Euler approximation of the solution of stochastic differential equation with time step size $\Delta t$, till $N^{\prime} \Delta t \leq r$, where $N^{\prime}$ is the unique number such that $N^{\prime} \Delta t \leq r$ and $\left(N^{\prime}+1\right) \Delta t>r$. If $N^{\prime} \Delta t<r$, define

$$
\begin{aligned}
\widehat{X}(r, 0, \omega)= & \widehat{X}\left(N^{\prime} \Delta t, 0, \omega\right)+f\left(N^{\prime} \Delta t, \widehat{X}\left(N^{\prime} \Delta t, 0, \omega\right)\right)\left(r-N^{\prime} \Delta t\right) \\
& +g\left(N^{\prime} \Delta t, \widehat{X}\left(N^{\prime} \Delta t, 0, \omega\right)\right)\left(W_{r}-W_{N^{\prime} \Delta t}\right) .
\end{aligned}
$$

Lemma 3.3. (Continuity of the discrete semi-flow with respect to the initial value) Denote by $\widetilde{X}_{r}^{0}$ and $\widetilde{Y}_{r}^{0}$ the solutions of the finite time Euler scheme with the initial values $\widetilde{\xi}$ and $\widetilde{\eta}$ at time 0 . Assume Conditions (A), (1) and Condition (2) for both initial values. Let $\Delta t$ be sufficiently small, $p \geq 1$. Then for any $\varepsilon>0$, there exists a $\delta>0$ such that for any $\|\widetilde{\xi}-\widetilde{\eta}\|_{p}<\delta$, we have

$$
\left\|\widetilde{X}_{r}^{0}(\omega, \widetilde{\xi})-\widetilde{Y}_{r}^{0}(\omega, \widetilde{\eta})\right\|_{p}<\varepsilon .
$$

Proof. Note that $\widetilde{X}_{N^{\prime} \Delta t}^{0}$ and $\widetilde{Y}_{N^{\prime} \Delta t}^{0}$ satisfy analogues of (3.2), with initial value $\widetilde{\xi}$ and $\widetilde{\eta}$ at time 0 instead of $-k \tau$. Apply the Euler scheme on the finite time $r^{\prime}=N^{\prime} \Delta t$ to obtain

$$
\begin{aligned}
& \left|\widetilde{X}_{r^{\prime}}^{0}(\omega, \widetilde{\xi})-\widetilde{Y}_{r^{\prime}}^{0}(\omega, \widetilde{\eta})\right|^{p} \\
& \quad \leq 3^{p-1}\left|(I+A \Delta t)^{p N^{\prime}}\right||\widetilde{\xi}-\widetilde{\eta}|^{p}+3^{p-1}(\Delta t)^{p}\left|(I+A \Delta t)^{p N^{\prime}}\right|\left|\sum_{i=0}^{N^{\prime}-1}(I+A \Delta t)^{-i-1} \widetilde{F}_{i}\right|^{p} \\
& \quad+3^{p-1}\left|(I+A \Delta t)^{p N^{\prime}}\right|\left|\sum_{i=0}^{N^{\prime}-1}(I+A \Delta t)^{-i-1} \widetilde{G}_{i}\left(W_{(i+1) \Delta t}-W_{i \Delta t}\right)\right|^{p},
\end{aligned}
$$

where $\widetilde{F}_{i}:=f\left(i \Delta t, \widetilde{X}_{i \Delta t}^{0}\right)-f\left(i \Delta t, \widetilde{Y}_{i \Delta t}^{0}\right), \widetilde{G}_{i}:=g\left(i \Delta t, \widetilde{X}_{i \Delta t}^{0}\right)-g\left(i \Delta t, \widetilde{Y}_{i \Delta t}^{0}\right)$. Denote $\widetilde{\zeta}_{i}:=\widetilde{X}_{i \Delta t}^{0}-\widetilde{Y}_{i \Delta t}^{0}$. For convenience, we denote $C_{p}=3^{p-1}, C_{p, N^{\prime}}=3^{p-1} N^{\prime p-1}$. Taking expectation on both sides of (3.9), and noting the Lipschitz condition of function $f$ and $g$, we have

$$
\begin{aligned}
(1-\alpha \Delta t)^{-p N^{\prime}}\left\|\widetilde{\zeta}_{N^{\prime}}\right\|_{p}^{p} \leq & C_{p}\|\widetilde{\xi}-\widetilde{\eta}\|_{p}^{p}+C_{p, N^{\prime}}(\Delta t)^{p} \sum_{i=0}^{N^{\prime}-1}(1-\alpha \Delta t)^{-(i+1) p} \beta_{1}^{p}\left\|\widetilde{\zeta}_{i}\right\|_{p}^{p} \\
& +C_{p, N^{\prime}}(\Delta t)^{p / 2} \sum_{i=0}^{N^{\prime}-1}(1-\alpha \Delta t)^{-(i+1) p} \beta_{2}^{p}\left\|\widetilde{\zeta}_{i}\right\|_{p}^{p} \\
= & C_{p}\|\widetilde{\xi}-\widetilde{\eta}\|_{p}^{p}+\widetilde{K} \sum_{i=0}^{N^{\prime}-1}(1-\alpha \Delta t)^{-i p}\left\|\widetilde{\zeta}_{i}\right\|_{p}^{p}
\end{aligned}
$$


where $\widetilde{K}=\frac{C_{p, N^{\prime}}\left((\Delta t)^{p} \beta_{1}^{p}+(\Delta t)^{p / 2} \beta_{2}^{p}\right)}{(1-\alpha \Delta t)^{p}}$, which is bounded for any $1 \leq p<+\infty$. Then by the Gronwall inequality, we have $\left\|\widetilde{\zeta}_{N^{\prime}}\right\|_{p}^{p} \leq C_{p}\|\widetilde{\xi}-\widetilde{\eta}\|_{p}^{p}\left((1+\widetilde{K})(1-\alpha \Delta t)^{p}\right)^{N^{\prime}}$. Note $(1+\widetilde{K})(1-\alpha \Delta t)^{p} \leq(1-$ $\alpha \Delta t)^{p}+C_{p, N^{\prime}}\left((\Delta t)^{p} \beta_{1}^{p}+(\Delta t)^{p / 2} \beta_{2}^{p}\right) \leq 1+C_{p, N^{\prime}}$. Result (3.8) at $r^{\prime}=N^{\prime} \Delta t$ follows by taking $\delta=$ $\frac{\varepsilon}{C_{p}}\left(1+C_{p, N^{\prime}}\right)^{-N^{\prime}}$. Finally, (3.8) at time $r$ follows from (3.7) and the estimate at $r^{\prime}=N^{\prime} \Delta t$.

Theorem 3.4. Assume that Condition (1) and $\Delta t$ is fixed and small enough. The time domain is divided as $\tau=n \Delta t$. Then there exists $\widehat{X}_{r}^{*} \in L^{2}(\Omega)$ such that for any initial values $\xi$ satisfying Condition (2), the solution of the Euler-Maruyama scheme satisfies

$$
\lim _{k \rightarrow \infty}\left\|\widehat{X}_{r}^{-k \tau}(\xi)-\widehat{X}_{r}^{*}\right\|_{2}=0
$$

and $\widehat{X}_{r}^{*}$ satisfies the random periodicity property.

Proof. Firstly, we note that the proof of the convergence of the process $\widehat{X}_{0}^{-k \tau}$ can be made similarly as that of Theorem 2.4. According to Lemma 3.1 we know that for any $M$, we have $\widehat{X}_{-k \tau+M \Delta t}^{-k \tau} \in L^{2}(\Omega)$. We use a similar construction of a Cauchy sequence as in Theorem 2.4. As we assume that $\tau=n \Delta t$ and $k \tau=k n \Delta t=: N \Delta t$, we have the following result by using semi-flow property, for any $m \geq 1$,

$$
\widehat{X}_{0}^{-(k+m) \tau}=\widehat{X}_{0}^{-(N+m n) \Delta t}=\widehat{X}_{0}^{-N \Delta t} \circ \widehat{X}_{-N \Delta t}^{-(N+m n) \Delta t} .
$$

It is a same process as $\widehat{X}_{0}^{-N \Delta t}$ with a different initial value. By Lemma 3.2 we have that for any $\varepsilon>0$ there exists $N^{*}$ such that for any $N \geq N^{*}, \Delta t>0$, we have

$$
\left\|\widehat{X}_{0}^{-k \tau}-\widehat{X}_{0}^{-(k+m) \tau}\right\|_{2}=\left\|\widehat{X}_{0}^{-N \Delta t}-\widehat{X}_{0}^{-(N+m n) \Delta t}\right\|_{2}<\varepsilon .
$$

Then we construct the Cauchy sequence $\widehat{X}_{i}=\widehat{X}_{0}^{-i \tau}$, which converges to some $\widehat{X}^{*}$ in $L^{2}(\Omega)$. We now use the same method to prove the convergence is independent of the initial point. Note for fixed $\Delta t$,

$$
\left\|\widehat{X}^{*}-\widehat{X}_{0}^{-k \tau}(\eta)\right\|_{2} \leq\left\|\widehat{X}^{*}-\widehat{X}_{0}^{-k \tau}(\xi)\right\|_{2}+\left\|\widehat{X}_{0}^{-k \tau}(\xi)-\widehat{X}_{0}^{-k \tau}(\eta)\right\|_{2} \stackrel{N \rightarrow \infty}{\longrightarrow} 0,
$$

where $N \rightarrow \infty$ is equivalent to $k \rightarrow \infty$.

Define $\widehat{X}^{*}(r, \omega):=\widehat{X}(r, 0, \omega) \circ \widehat{X}^{*}, r \geq 0$. According to Lemma 3.3, we have

$$
\widehat{X}_{r}^{-k \tau}(\omega)=\widehat{X}(r, 0, \omega) \circ \widehat{X}_{0}^{-k \tau}(\omega) \underset{L^{2}(\Omega)}{\stackrel{k \rightarrow \infty}{\longrightarrow}} \widehat{X}(r, 0, \omega) \circ \widehat{X}^{*}(\omega)=\widehat{X}^{*}(r, \omega),
$$

so (3.10) holds. On the other hand, similar to the proof of (2.3), we obtain

$$
\widehat{X}_{r+\tau}^{\tau}(\omega, \xi(\omega))=\widehat{X}_{r}^{0}\left(\theta_{\tau} \omega, \xi\left(\theta_{\tau} \omega\right)\right)=\theta_{\tau} \widehat{X}_{r}^{0}(\omega, \xi(\omega)) .
$$

Therefore,

$$
\widehat{X}_{r}^{-k \tau}\left(\theta_{\tau} \omega\right)=\widehat{X}\left(r, 0, \theta_{\tau} \omega\right) \circ \widehat{X}_{0}^{-k \tau}\left(\theta_{\tau} \omega\right) \underset{L^{2}(\Omega)}{\stackrel{k \rightarrow \infty}{\longrightarrow}} \widehat{X}\left(r, 0, \theta_{\tau} \omega\right) \circ \widehat{X}^{*}\left(\theta_{\tau} \omega\right)=\widehat{X}^{*}\left(r, \theta_{\tau} \omega\right) .
$$

But,

$$
\widehat{X}_{r+\tau}^{-k \tau+\tau}(\omega) \underset{L^{2}(\Omega)}{\stackrel{k \rightarrow \infty}{\longrightarrow}} \widehat{X}^{*}(r+\tau, \omega), \text { and } \widehat{X}_{r+\tau}^{-k \tau+\tau}(\omega)=\widehat{X}_{r}^{-k \tau}\left(\theta_{\tau} \omega\right), \mathbb{P}-\text { a.s }
$$

thus, we have $\widehat{X}^{*}(r+\tau, \omega)=\widehat{X}^{*}\left(r, \theta_{\tau} \omega\right), \mathbb{P}-$ a.s.

Example 3.5. Consider a specific SDE

$$
d X_{t}^{t_{0}}=-\pi X_{t}^{t_{0}} d t+\sin (\pi t) d t+X_{t}^{t_{0}} d W_{t}
$$

According to Theorem 2.4, (3.11) has a random periodic solution. By Theorem 3.4, its Euler-Maruyama dissertation also has a random periodic path. To see the "periodicity" numerically, we provided two 

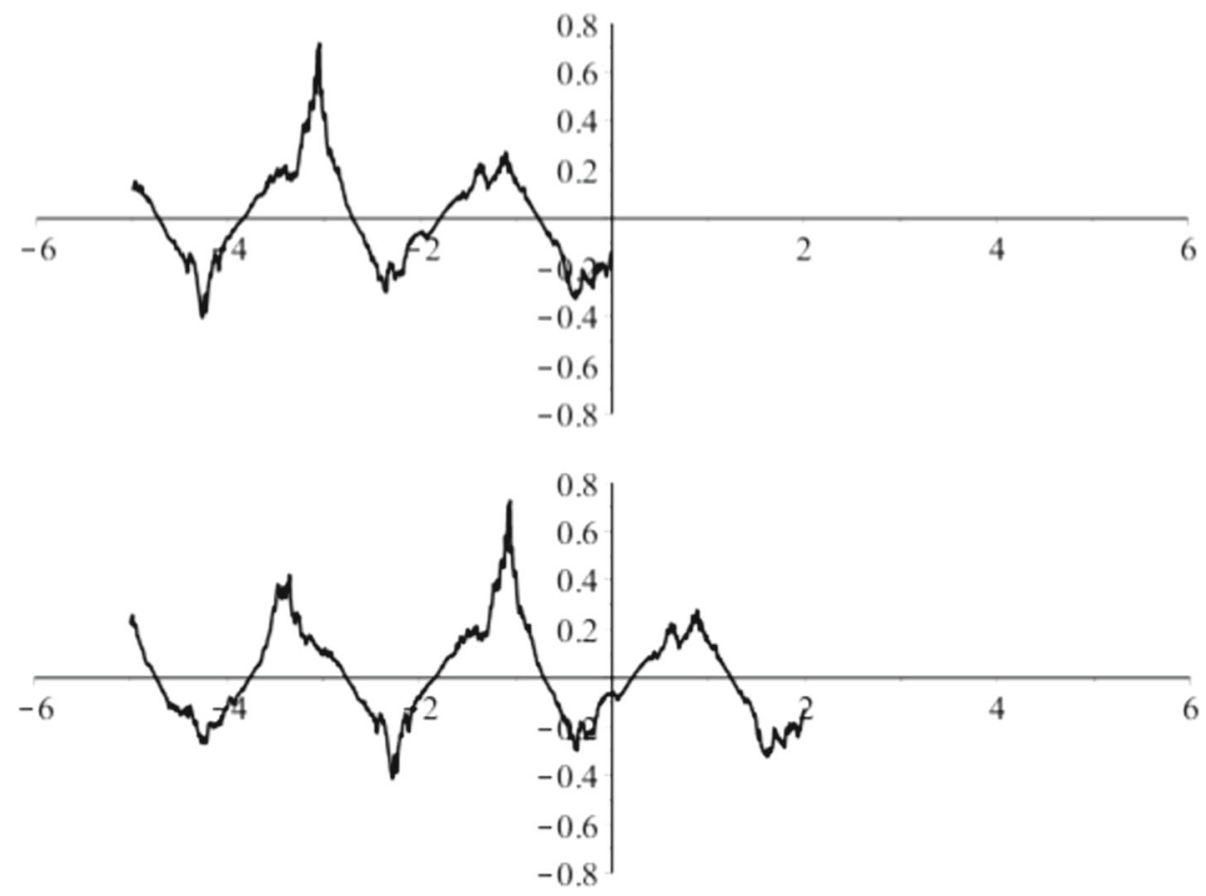

FIG. 1. Simulations of the processes $\left\{\hat{X}_{t}^{*}(\omega),-5 \leq t \leq 0\right\}$ and $\left\{\hat{X}_{t}^{*}\left(\theta_{-2} \omega\right),-5 \leq t \leq 2\right\}$

methods. One approach is to simulate the processes $\hat{X}_{t}^{*}(\omega)=\widehat{X}_{t}^{-6}(\omega, 0.5),-5 \leq t \leq 0$, and $\hat{X}_{t}^{*}\left(\theta_{-2} \omega\right)=$ $\widehat{X}_{t}^{-6}\left(\theta_{-2} \omega, 0.5\right),-5 \leq t \leq 2$, with the same $\omega$ and step size $\Delta t=0.01$ (Fig. 1). One can see that these two trajectories exactly repeat each with a time shift of one period (only comparing the graph of $\hat{X}_{t}^{*}\left(\theta_{-2} \omega\right)$ for $-3 \leq t \leq 2)$. The second method is the simulation of $\left\{\hat{X}_{t}^{*}\left(\theta_{-t} \omega\right), 0 \leq t \leq 6\right\}$ for the same realisation $\omega$ and step size as before (Fig. 2). One can easily see that Fig. 2 is a perfect periodic curve. This agrees with the fact that if $\hat{X}_{t}^{*}(\omega)$ is a random periodic path iff $\hat{X}_{t}^{*}\left(\theta_{-t} \omega\right)$ is periodic, i.e. $\hat{X}_{t+\tau}^{*}\left(\theta_{-(t+\tau)} \omega\right)=\hat{X}_{t}^{*}\left(\theta_{-t} \omega\right)$. Note in theory $\hat{X}_{t}^{*}=\hat{X}_{t}^{-\infty}$, but we take pull-back time -6 as this is already enough to generate a good convergence to the random periodic paths $\hat{X}_{t}^{*}(\cdot)$ for $t \geq-5$ by the solution starting at -6 from 0.5 for both cases. The choice of the initial position does not affect random periodic paths, but the time to take for the convergence.

\subsection{Modified Milstein scheme}

We will consider the Milstein scheme which will increase the convergence order for the infinite horizon problem.

Condition $\left(\mathbf{1}^{\prime}\right)$. Assume there exists a constant $\tau>0$ such that for any $t \in \mathbb{R}, x \in \mathbb{R}^{m}, f(t+\tau, x)=$ $f(t, x), g(t+\tau, x)=g(t, x)$, and there exist constants $C_{0}, \beta_{1}, \beta_{2}>0$ with $\beta_{1}+\frac{\beta_{2}^{2}}{2}<\left|\lambda_{1}\right|$ such that for any $s, t \in \mathbb{R}$ and $x \in \mathbb{R}^{m}$,

$$
\begin{gathered}
|f(s, x)-f(t, y)| \leq C_{0}|s-t|+\beta_{1}|x-y|, \\
|g(s, x)-g(t, y)| \leq C_{0}|s-t|+\beta_{2}|x-y| .
\end{gathered}
$$

Meanwhile, we assume the boundedness of first-order partial derivative of function $f$ and $g$ with respect to $x$. 


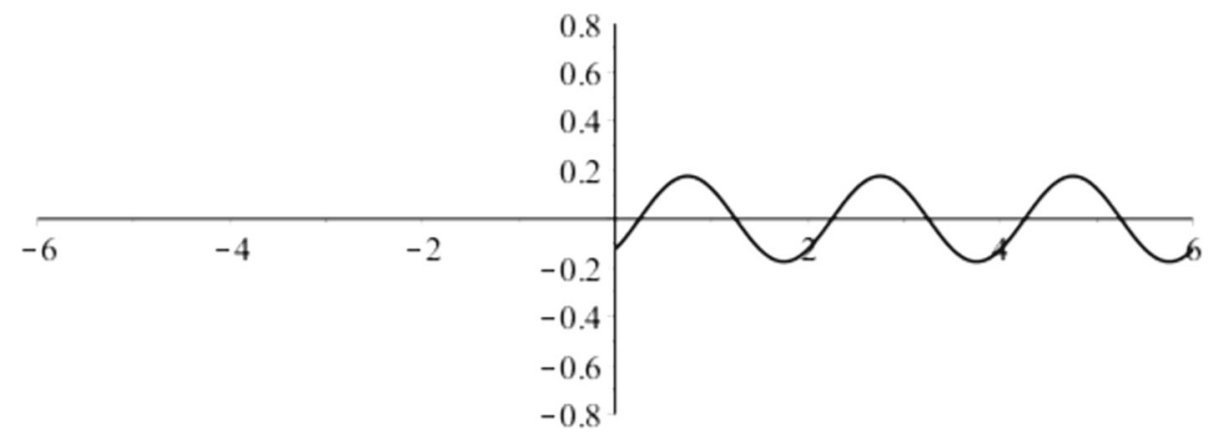

FIG. 2. Simulation of the process $\left\{\hat{X}_{t}^{*}\left(\theta_{-t} \omega\right), 0 \leq t \leq 6\right\}$

The iteration formula for the modified SRK scheme is

$$
\begin{aligned}
\widehat{X}_{-k \tau+(i+1) \Delta t}^{-k \tau}= & \widehat{X}_{-k \tau+i \Delta t}^{-k \tau}+A \widehat{X}_{-k \tau+i \Delta t}^{-k \tau} \Delta t+f\left(i \Delta t, \widehat{X}_{-k \tau+i \Delta t}^{-k \tau}\right) \Delta t \\
& +g\left(i \Delta t, \widehat{X}_{-k \tau+i \Delta t}^{-k \tau}\right)\left(\Delta W_{i}\right) \\
& \left.+\frac{\Delta Z_{i}}{2 \sqrt{\Delta t}}\left[f\left(i \Delta t, \widehat{\Upsilon}_{+}\left(\widehat{X}_{-k \tau+i \Delta t}^{-k \tau}\right)\right)\right)-f\left(i \Delta t, \widehat{\Upsilon}_{-}\left(\widehat{X}_{-k \tau+i \Delta t}^{-k \tau}\right)\right)\right] \\
& \left.+\frac{\left(\Delta W_{i}\right)^{2}-\Delta t}{4 \sqrt{\Delta t}}\left[g\left(i \Delta t, \widehat{\Upsilon}_{+}\left(\widehat{X}_{-k \tau+i \Delta t}^{-k \tau}\right)\right)\right)-g\left(i \Delta t, \widehat{\Upsilon}_{-}\left(\widehat{X}_{-k \tau+i \Delta t}^{-k \tau}\right)\right)\right],
\end{aligned}
$$

with

$$
\begin{aligned}
\widehat{\Upsilon}_{ \pm}\left(\widehat{X}_{-k \tau+i \Delta t}^{-k \tau}\right)= & \widehat{X}_{-k \tau+i \Delta t}^{-k \tau}+A \widehat{X}_{-k \tau+i \Delta t}^{-k \tau} \Delta t+f\left(i \Delta t, \widehat{X}_{-k \tau+i \Delta t}^{-k \tau}\right) \Delta t \\
& \pm g\left(i \Delta t, \widehat{X}_{-k \tau+i \Delta t}^{-k \tau}\right) \sqrt{\Delta t}
\end{aligned}
$$

and

$$
\begin{aligned}
\Delta W_{i} & =\int_{-k \tau+i \Delta t}^{-k \tau+(i+1) \Delta t} d W_{s}=W_{-k \tau+(i+1) \Delta t}-W_{-k \tau+i \Delta t}, \\
\Delta Z_{i} & =\int_{-k \tau+i \Delta t}^{-k \tau+(i+1) \Delta t} \int_{-k \tau+i \Delta t}^{s} d W_{u} d s
\end{aligned}
$$

where $i=0,1,2, \ldots$, and $\widehat{X}_{-k \tau+0 \Delta t}^{-k \tau}=\xi$. Here we used the approximation of $\Delta Z_{i}$ by the method of Kloeden and Platen in [14].

Theorem 3.6. Assume that Conditions $(A),\left(1^{\prime}\right)$ hold and $\Delta t$ is fixed and small enough. The time domain is divided as $\tau=n \Delta t$. Then there exists $\widehat{X}_{r}^{*} \in L^{2}(\Omega)$ such that for any initial values $\xi$ satisfying Condition (2), the solution of the Milstein scheme satisfies

$$
\lim _{k \rightarrow \infty}\left\|\widehat{X}_{r}^{-k \tau}(\xi)-\widehat{X}_{r}^{*}\right\|_{2}=0
$$

and $\widehat{X}_{r}^{*}$ satisfies the random periodicity property.

Proof. The proof is by a similar argument as Theorem 3.4. As it is tedious and there is no special difficulty, so omitted here. 
Remark 3.7. For the Milstein scheme, the existence of constants as $\widehat{K}_{1}, \widehat{K}_{2}, \widehat{K}_{3}, \widehat{K}_{4}$ in the proof of EulerMaruyama scheme is guaranteed by the boundedness of partial derivatives of functions $f$ and $g$. Then we still have the convergence for different initial values and the boundedness of the discrete processes. The addition term $\left.\frac{\Delta Z_{i}}{2 \sqrt{\Delta t}}\left[f\left(i \Delta t, \widehat{\Upsilon}_{+}\left(\widehat{X}_{-k \tau+i \Delta t}^{-k \tau}\right)\right)\right)-f\left(i \Delta t, \widehat{\Upsilon}_{-}\left(\widehat{X}_{-k \tau+i \Delta t}^{-k \tau}\right)\right)\right]$ in the scheme does not influence the result of the convergence. However, when we analyse the error between approximation and the exact solution of random periodic solutions, this term is necessary for infinite horizon case to satisfy the order of error.

\section{The error estimate}

\subsection{Euler-Maruyama method}

In the last two sections, we proved the existence of random periodic solutions of SDE (1.3) and its discretisations as the limits of semi-flows when the starting times were pushed to $-\infty$. The next step is to estimate the error between these two limits. Now we need to consider the difference between the discrete approximate solution and the exact solution. The exact solution at time $-k \tau+M \Delta t$ is as follows

$$
\begin{aligned}
X_{-k \tau+M \Delta t}^{-k \tau}(\omega, \xi)= & e^{A M \Delta t} \xi+e^{A(M \Delta t-k \tau)} \int_{-k \tau}^{M \Delta t-k \tau} e^{-A s} f\left(s, X_{s}^{-k \tau}\right) d s \\
& +e^{A(M \Delta t-k \tau)} \int_{-k \tau}^{M \Delta t-k \tau} e^{-A s} g\left(s, X_{s}^{-k \tau}\right) d W_{s} .
\end{aligned}
$$

Lemma 4.1. Assume Conditions (A), (1) and (2). Choose $\Delta t=\tau / n$ for some $n \in \mathbb{N}$ and $N=k n$. Then there exists a constant $K>0$ such that for any sufficiently small fixed $\Delta t$ and $N^{\prime} \in \mathbb{N}$, we have

$$
\limsup _{k \rightarrow \infty}\left\|X_{N^{\prime} \Delta t}^{-k \tau}-\widehat{X}_{N^{\prime} \Delta t}^{-k \tau}\right\|_{2} \leq K \sqrt{\Delta t}
$$

where $X_{N^{\prime} \Delta t}^{-k \tau}$ and $\widehat{X}_{N^{\prime} \Delta t}^{-k \tau}$ are the exact and the numerical solutions given by (4.1) and (3.2), respectively, $K$ is independent of $N^{\prime}$ and $\Delta t$.

Proof. In the following proof, we always denote by $\hat{K}$. the constant derived from the underlining computation unless otherwise stated. For any $M \in \mathbb{N}$, we have

$$
\begin{aligned}
& X_{-k \tau+M \Delta t}^{-k \tau}-\widehat{X}_{-k \tau+M \Delta t}^{-k \tau}=\left(e^{A M \Delta t}-(I+A \Delta t)^{M}\right) \xi+e^{A(M \Delta t-k \tau)} \int_{-k \tau}^{M \Delta t-k \tau} e^{-A s} f\left(s, X_{s}^{-k \tau}\right) d s \\
& -\sum_{i=0}^{M-1}(I+A \Delta t)^{M-i-1} f\left(i \Delta t, \widehat{X}_{-k \tau+i \Delta t}^{-k \tau}\right) \Delta t+e^{A(M \Delta t-k \tau)} \int_{-k \tau}^{M \Delta t-k \tau} e^{-A s} g\left(s, X_{s}^{-k \tau}\right) d W_{s} \\
& -\sum_{i=0}^{M-1}(I+A \Delta t)^{M-i-1} g\left(i \Delta t, \widehat{X}_{-k \tau+i \Delta t}^{-k \tau}\right)\left(W_{-k \tau+(i+1) \Delta t}-W_{-k \tau+i \Delta t}\right) .
\end{aligned}
$$

Similar to the method of Lemma 3.1, firstly consider 


$$
\begin{aligned}
& (1-\alpha \Delta t)^{-2 M}\left|X_{-k \tau+M \Delta t}^{-k \tau}-\widehat{X}_{-k \tau+M \Delta t}^{-k \tau}\right|^{2} \\
& \quad=\sum_{i=0}^{M-1}(1-\alpha \Delta t)^{-2 i}\left(\frac{\left|X_{-k \tau+(i+1) \Delta t}^{-k \tau}-\widehat{X}_{-k \tau+(i+1) \Delta t}^{-k \tau}\right|^{2}}{(1-\alpha \Delta t)^{2}}-\left|X_{-k \tau+i \Delta t}^{-k \tau}-\widehat{X}_{-k \tau+i \Delta t}^{-k \tau}\right|^{2}\right)
\end{aligned}
$$

For simplicity we denote

$$
\begin{aligned}
& B_{1}=\frac{1}{1-\alpha \Delta t} \int_{i \Delta t-k \tau}^{(i+1) \Delta t-k \tau}\left(e^{-A(s+k \tau-(i+1) \Delta t)} f\left(s, X_{s}^{-k \tau}\right)-f\left(i \Delta t, \widehat{X}_{-k \tau+i \Delta t}^{-k \tau}\right)\right) d s, \\
& B_{2}=\frac{1}{1-\alpha \Delta t} \int_{i \Delta t-k \tau}^{(i+1) \Delta t-k \tau}\left(e^{-A(s+k \tau-(i+1) \Delta t)} g\left(s, X_{s}^{-k \tau}\right)-g\left(i \Delta t, \widehat{X}_{-k \tau+i \Delta t}^{-k \tau}\right)\right) d W_{s} .
\end{aligned}
$$

Therefore,

$$
\begin{aligned}
& X_{-k \tau+(i+1) \Delta t}^{-k \tau}-\widehat{X}_{-k \tau+(i+1) \Delta t}^{-k \tau} \\
& \quad=e^{A \Delta t} X_{-k \tau+i \Delta t}^{-k \tau}-(I+A \Delta t) \widehat{X}_{-k \tau+i \Delta t}^{-k \tau}+(1-\alpha \Delta t)\left(B_{1}+B_{2}\right) .
\end{aligned}
$$

Now we consider

$$
\begin{aligned}
& \frac{\left|X_{-k \tau+(i+1) \Delta t}^{-k \tau}-\widehat{X}_{-k \tau+(i+1) \Delta t}^{-k \tau}\right|^{2}}{(1-\alpha \Delta t)^{2}}-\left|X_{-k \tau+i \Delta t}^{-k \tau}-\widehat{X}_{-k \tau+i \Delta t}^{-k \tau}\right|^{2} \\
& =\left(X_{-k \tau+i \Delta t}^{-k \tau}-\widehat{X}_{-k \tau+i \Delta t}^{-k \tau}\right)^{T}\left(\frac{e^{A \Delta t}}{1-\alpha \Delta t}-I\right)\left(\frac{e^{A \Delta t}}{1-\alpha \Delta t}+I\right)\left(X_{-k \tau+i \Delta t}^{-k \tau}-\widehat{X}_{-k \tau+i \Delta t}^{-k \tau}\right) \\
& \quad+\left(\widehat{X}_{-k \tau+i \Delta t}^{-k \tau}\right)^{T}\left(\frac{e^{A \Delta t}-I-A \Delta t}{1-\alpha \Delta t}\right)^{2}\left(\widehat{X}_{-k \tau+i \Delta t}^{-k \tau}\right)+B_{1}^{T} B_{1}+B_{2}^{T} B_{2} \\
& \quad+2\left(X_{-k \tau+i \Delta t}^{-k \tau}-\widehat{X}_{-k \tau+i \Delta t}^{-k \tau}\right)^{T}\left(\frac{e^{A \Delta t}}{1-\alpha \Delta t}\right)\left(\frac{e^{A \Delta t}-I-A \Delta t}{1-\alpha \Delta t}\right)\left(\widehat{X}_{-k \tau+i \Delta t}^{-k \tau}\right) \\
& \quad+2\left(\left(X_{-k \tau+i \Delta t}^{-k \tau}\right)^{T}\left(\frac{e^{A \Delta t}}{1-\alpha \Delta t}\right)-\left(\widehat{X}_{-k \tau+i \Delta t}^{-k \tau}\right)^{T}\left(\frac{I+A \Delta t}{1-\alpha \Delta t}\right)\right) B_{1} \\
& \quad+2\left(\left(X_{-k \tau+i \Delta t}^{-k \tau}\right)^{T}\left(\frac{e^{A \Delta t}}{1-\alpha \Delta t}\right)-\left(\widehat{X}_{-k \tau+i \Delta t}^{-k \tau}\right)^{T}\left(\frac{I+A \Delta t}{1-\alpha \Delta t}\right)\right) B_{2}+2 B_{1}^{T} B_{2} .
\end{aligned}
$$

We note that the matrix $\left(\frac{e^{A \Delta t}}{1-\alpha \Delta t}-I\right)\left(\frac{e^{A \Delta t}}{1-\alpha \Delta t}+I\right)$ can be non-positive definite when we choose the $\Delta t$ small enough. Now we consider each term in (4.3). First,

$$
\begin{aligned}
& \mathbb{E}\left[\left(\widehat{X}_{-k \tau+i \Delta t}^{-k \tau}\right)^{T}\left(\frac{e^{A \Delta t}-I-A \Delta t}{1-\alpha \Delta t}\right)^{2} \widehat{X}_{-k \tau+i \Delta t}^{-k \tau}\right] \\
& \leq\left\|\widehat{X}_{-k \tau+i \Delta t}^{-k \tau}\right\|_{2}\left|\frac{\frac{1}{2} A^{2}(\Delta t)^{2}}{1-\alpha \Delta t}\right|^{2}\left\|\widehat{X}_{-k \tau+i \Delta t}^{-k \tau}\right\|_{2} \leq \widehat{K}_{5}(\Delta t)^{4}
\end{aligned}
$$


Next,

$$
\begin{aligned}
\mathbb{E}\left[B_{1}^{T} B_{1}\right]= & \mathbb{E}\left|B_{1}\right|^{2} \leq \frac{2(1+\mu)}{\mu(1-\alpha \Delta t)^{2}}\left(\int_{i \Delta t-k \tau}^{(i+1) \Delta t-k \tau}\left|e^{-A(s+k \tau-(i+1) \Delta t)}-I\right|\left\|f\left(s, X_{s}^{-k \tau}\right)\right\|_{2} d s\right)^{2} \\
& +\frac{2(1+\mu)}{\mu(1-\alpha \Delta t)^{2}}\left(\int_{i \Delta t-k \tau}^{(i+1) \Delta t-k \tau}\left\|f\left(s, X_{s}^{-k \tau}\right)-f\left(i \Delta t, X_{-k \tau+i \Delta t}^{-k \tau}\right)\right\|_{2} d s\right)^{2} \\
& +\frac{1+\mu}{(1-\alpha \Delta t)^{2}}\left(\int_{i \Delta t-k \tau}^{(i+1) \Delta t-k \tau}\left\|f\left(i \Delta t, X_{-k \tau+i \Delta t}^{-k \tau}\right)-f\left(i \Delta t, \widehat{X}_{-k \tau+i \Delta t}^{-k \tau}\right)\right\|_{2} d s\right)^{2}
\end{aligned}
$$

where $\mu$ is a small number from Young's inequality, which will be fixed later. By linear growth property of $f$ and Lemma 2.1, we know that $\left\|f\left(s, X_{s}^{-k \tau}\right)\right\|_{2}$ is bounded. So for the first term in (4.4) we only need to estimate

$$
\int_{i \Delta t-k \tau}^{(i+1) \Delta t-k \tau}\left|e^{-A(s+k \tau-(i+1) \Delta t)}-I\right| d s \leq \frac{(\Delta t)^{2}}{2} \operatorname{Tr}(-A) .
$$

By Condition (1) and Lemma 2.2, the second term in (4.4) becomes

$$
\begin{aligned}
& \quad \int_{i \Delta t-k \tau}^{(i+1) \Delta t-k \tau}\left\|f\left(s, X_{s}^{-k \tau}\right)-f\left(i \Delta t, X_{-k \tau+i \Delta t}^{-k \tau}\right)\right\|_{2} d s \\
& \leq \int_{i \Delta t-k \tau}^{(i+1) \Delta t-k \tau}\left(\left\|f\left(s, X_{s}^{-k \tau}\right)-f\left(i \Delta t, X_{s}^{-k \tau}\right)\right\|_{2}\right. \\
& \left.\quad+\left\|f\left(i \Delta t, X_{s}^{-k \tau}\right)-f\left(i \Delta t, X_{-k \tau+i \Delta t}^{-k \tau}\right)\right\|_{2}\right) d s \\
& \leq \int_{(i+1) \Delta t-k \tau}^{(i+1) \Delta t-k \tau} \int_{i \Delta t-k \tau} \beta_{0}|s-i \Delta t+k \tau|^{1 / 2} d s+X_{s}^{-k \tau}-X_{-k \tau+i \Delta t}^{-k \tau} \|_{2} d s \\
& \leq \int_{(i+1) \Delta t-k \tau}\left(C_{0}+\beta_{1} C_{4}\right) \sqrt{s-i \Delta t+k \tau} d s \\
& \leq \widehat{K}_{6}(\Delta t)^{\frac{3}{2}} .
\end{aligned}
$$

Applying the global Lipschitz condition, the third term of (4.4) becomes

$$
\begin{aligned}
& \int_{i \Delta t-k \tau}^{(i+1) \Delta t-k \tau}\left\|f\left(i \Delta t, X_{-k \tau+i \Delta t}^{-k \tau}\right)-f\left(i \Delta t, \widehat{X}_{-k \tau+i \Delta t}^{-k \tau}\right)\right\|_{2} d s \\
& \quad \leq \beta_{1} \Delta t\left\|X_{-k \tau+i \Delta t}^{-k \tau}-\widehat{X}_{-k \tau+i \Delta t}^{-k \tau}\right\|_{2} .
\end{aligned}
$$


We summarise the above inequalities to have

$$
\mathbb{E}\left[B_{1}^{T} B_{1}\right] \leq \widehat{K}_{7}(\Delta t)^{3}+\frac{(1+\mu) \beta_{1}^{2}(\Delta t)^{2}}{(1-\alpha \Delta t)^{2}}\left\|X_{-k \tau+i \Delta t}^{-k \tau}-\widehat{X}_{-k \tau+i \Delta t}^{-k \tau}\right\|_{2}^{2} .
$$

This term is of the third order of $\Delta t$ and second order of $\Delta t$ with $\left\|X_{-k \tau+i \Delta t}^{-k \tau}-\widehat{X}_{-k \tau+i \Delta t}^{-k \tau}\right\|_{2}^{2}$.

Similar to the $\mathbb{E}\left[B_{1}^{T} B_{1}\right]$, the following term can be estimated as

$$
\begin{aligned}
\mathbb{E}\left[B_{2}^{T} B_{2}\right]=\mathbb{E}\left|B_{2}\right|^{2} & \leq \frac{2(1+\mu)}{\mu(1-\alpha \Delta t)^{2}} \int_{i \Delta t-k \tau}^{(i+1) \Delta t-k \tau}\left|e^{-A(s+k \tau-(i+1) \Delta t)}-I\right|^{2}\left\|g\left(s, X_{s}^{-k \tau}\right)\right\|_{2}^{2} d s \\
& +\frac{2(1+\mu)}{\mu(1-\alpha \Delta t)^{2}} \int_{i \Delta t-k \tau}^{(i+1) \Delta t-k \tau}\left\|g\left(s, X_{s}^{-k \tau}\right)-g\left(i \Delta t, X_{-k \tau+i \Delta t}^{-k \tau}\right)\right\|_{2}^{2} d s \\
& +\frac{1+\mu}{(1-\alpha \Delta t)^{2}} \int_{i \Delta t-k \tau}^{(i+1) \Delta t-k \tau}\left\|g\left(i \Delta t, X_{-k \tau+i \Delta t}^{-k \tau}\right)-g\left(i \Delta t, \widehat{X}_{-k \tau+i \Delta t}^{-k \tau}\right)\right\|_{2}^{2} d s
\end{aligned}
$$

where $\mu$ is a small number from Young's inequality, which will be fixed later. By the linear growth property of $g$ and Lemma 2.1, we know that $\left\|g\left(s, X_{s}^{-k \tau}\right)\right\|_{2}^{2}$ is bounded. So we only need to estimate

$$
\int_{i \Delta t-k \tau}^{(i+1) \Delta t-k \tau}\left|e^{-A(s+k \tau-(i+1) \Delta t)}-I\right|^{2} d s \leq \frac{2}{3}(\Delta t)^{3} \operatorname{Tr}\left(A^{2}\right) .
$$

By Condition (1) and Lemma 2.2, the second term in (4.6) becomes

$$
\begin{aligned}
& \quad \int_{i \Delta t-k \tau}^{(i+1) \Delta t-k \tau}\left\|g\left(s, X_{s}^{-k \tau}\right)-g\left(i \Delta t, X_{-k \tau+i \Delta t}^{-k \tau}\right)\right\|_{2}^{2} d s \\
& \leq \int_{i \Delta t-k \tau}^{(i+1) \Delta t-k \tau} 2\left(C_{0}^{2}+\beta_{2}^{2} C_{4}^{2}\right)|s-i \Delta t+k \tau| d s \leq \widehat{K}_{8}(\Delta t)^{2} .
\end{aligned}
$$

The third term follows from the global Lipschitz condition

$$
\begin{aligned}
& \int_{i \Delta t-k \tau}^{(i+1) \Delta t-k \tau}\left\|g\left(i \Delta t, X_{-k \tau+i \Delta t}^{-k \tau}\right)-g\left(i \Delta t, \widehat{X}_{-k \tau+i \Delta t}^{-k \tau}\right)\right\|_{2}^{2} d s \\
& \quad \leq \beta_{2}^{2} \Delta t\left\|X_{-k \tau+i \Delta t}^{-k \tau}-\widehat{X}_{-k \tau+i \Delta t}^{-k \tau}\right\|_{2}^{2} .
\end{aligned}
$$

Conclude the above results to obtain

$$
\mathbb{E}\left[B_{2}^{T} B_{2}\right] \leq \widehat{K}_{9}(\Delta t)^{2}+\frac{(1+\mu) \beta_{2}^{2} \Delta t}{(1-\alpha \Delta t)^{2}}\left\|X_{-k \tau+i \Delta t}^{-k \tau}-\widehat{X}_{-k \tau+i \Delta t}^{-k \tau}\right\|_{2}^{2} .
$$


The fifth term of (4.3) can be estimate as follows

$$
\begin{aligned}
& \mathbb{E}\left[2\left(X_{-k \tau+i \Delta t}^{-k \tau}-\widehat{X}_{-k \tau+i \Delta t}^{-k \tau}\right)^{T}\left(\frac{e^{A \Delta t}}{1-\alpha \Delta t}\right)\left(\frac{e^{A \Delta t}-I-A \Delta t}{1-\alpha \Delta t}\right)\left(\widehat{X}_{-k \tau+i \Delta t}^{-k \tau}\right)\right] \\
& \quad \leq 2\left\|X_{-k \tau+i \Delta t}^{-k \tau}-\widehat{X}_{-k \tau+i \Delta t}^{-k \tau}\right\|_{2} \frac{\frac{1}{2}\left|A^{2}\right|(\Delta t)^{2}}{(1-\alpha \Delta t)^{2}}\left\|\widehat{X}_{-k \tau+i \Delta t}^{-k \tau}\right\|_{2} \\
& \quad \leq \widehat{K}_{10}(\Delta t)^{2}\left\|X_{-k \tau+i \Delta t}^{-k \tau}-\widehat{X}_{-k \tau+i \Delta t}^{-k \tau}\right\|_{2} .
\end{aligned}
$$

To estimate the sixth term of (4.3),

$$
\begin{aligned}
\mathbb{E} & {\left[2\left(\left(X_{-k \tau+i \Delta t}^{-k \tau}\right)^{T}\left(\frac{e^{A \Delta t}}{1-\alpha \Delta t}\right)-\left(\widehat{X}_{-k \tau+i \Delta t}^{-k \tau}\right)^{T}\left(\frac{I+A \Delta t}{1-\alpha \Delta t}\right)\right) B_{1}\right] } \\
= & \mathbb{E}\left[2\left(X_{-k \tau+i \Delta t}^{-k \tau}\right)^{T}\left(\frac{e^{A \Delta t}}{1-\alpha \Delta t}-\frac{I+A \Delta t}{1-\alpha \Delta t}\right) B_{1}\right] \\
& +\mathbb{E}\left[2\left(X_{-k \tau+i \Delta t}^{-k \tau}-\widehat{X}_{-k \tau+i \Delta t}^{-k \tau}\right)^{T}\left(\frac{I+A \Delta t}{1-\alpha \Delta t}\right) B_{1}\right] .
\end{aligned}
$$

Now we discuss these two terms separately,

$$
\begin{aligned}
& \mathbb{E}\left[2\left(X_{-k \tau+i \Delta t}^{-k \tau}\right)^{T}\left(\frac{e^{A \Delta t}}{1-\alpha \Delta t}-\frac{I+A \Delta t}{1-\alpha \Delta t}\right) B_{1}\right] \leq 2\left\|X_{-k \tau+i \Delta t}^{-k \tau}\right\|_{2} \frac{\left|\frac{1}{2} A^{2}(\Delta t)^{2}\right|}{1-\alpha \Delta t}\left\|B_{1}\right\|_{2} \\
& \leq \widehat{K}_{12}(\Delta t)^{7 / 2}+\frac{\sqrt{1+\mu} \beta_{1} \widehat{K}_{11}(\Delta t)^{3}}{(1-\alpha \Delta t)^{2}}\left\|X_{-k \tau+i \Delta t}^{-k \tau}-\widehat{X}_{-k \tau+i \Delta t}^{-k \tau}\right\|_{2} .
\end{aligned}
$$

And,

$$
\begin{aligned}
& \mathbb{E}\left[2\left(X_{-k \tau+i \Delta t}^{-k \tau}-\widehat{X}_{-k \tau+i \Delta t}^{-k \tau}\right)^{T}\left(\frac{I+A \Delta t}{1-\alpha \Delta t}\right) B_{1}\right] \\
& \leq \frac{2 \sqrt{\widehat{K}_{7}}(\Delta t)^{3 / 2}}{1-\alpha \Delta t}\left\|X_{-k \tau+i \Delta t}^{-k \tau}-\widehat{X}_{-k \tau+i \Delta t}^{-k \tau}\right\|_{2}(1+\Delta t|A|) \\
&+\frac{2 \sqrt{1+\mu} \beta_{1} \Delta t}{(1-\alpha \Delta t)^{2}}\left\|X_{-k \tau+i \Delta t}^{-k \tau}-\widehat{X}_{-k \tau+i \Delta t}^{-k \tau}\right\|_{2}^{2}(1+\Delta t|A|) .
\end{aligned}
$$

We use the conditional expectation to eliminate the seventh term

$$
\begin{aligned}
\mathbb{E} & {\left[\left(\left(X_{-k \tau+i \Delta t}^{-k \tau}\right)^{T}\left(\frac{e^{A \Delta t}}{1-\alpha \Delta t}\right)-\left(\widehat{X}_{-k \tau+i \Delta t}^{-k \tau}\right)^{T}\left(\frac{I+A \Delta t}{1-\alpha \Delta t}\right)\right) B_{2}\right] } \\
& =\mathbb{E}\left[\left(\left(X_{-k \tau+i \Delta t}^{-k \tau}\right)^{T}\left(\frac{e^{A \Delta t}}{1-\alpha \Delta t}\right)-\left(\widehat{X}_{-k \tau+i \Delta t}^{-k \tau}\right)^{T}\left(\frac{I+A \Delta t}{1-\alpha \Delta t}\right)\right) \mathbb{E}\left[B_{2} \mid \mathcal{F}^{i \Delta t-k \tau}\right]\right] \\
& =0 .
\end{aligned}
$$

For the last term,

$$
\begin{aligned}
\mathbb{E}\left[2 B_{1}^{T} B_{2}\right] & \leq 2\left\|B_{1}^{T}\right\|_{2} \cdot\left\|B_{2}\right\|_{2} \\
& \leq \widehat{K}_{13}(\Delta t)^{5 / 2}+\widehat{K}_{14}(\Delta t)^{3 / 2}\left\|X_{-k \tau+i \Delta t}^{-k \tau}-\widehat{X}_{-k \tau+i \Delta t}^{-k \tau}\right\|_{2}^{2}
\end{aligned}
$$


Combining all the estimation above, we have

$$
\begin{aligned}
& \frac{\left|X_{-k \tau+(i+1) \Delta t}^{-k \tau}-\widehat{X}_{-k \tau+(i+1) \Delta t}^{-k \tau}\right|^{2}}{(1-\alpha \Delta t)^{2}}-\left|X_{-k \tau+i \Delta t}^{-k \tau}-\widehat{X}_{-k \tau+i \Delta t}^{-k \tau}\right|^{2} \\
& \leq\left(\frac{(1+\mu) \beta_{2}^{2} \Delta t}{(1-\alpha \Delta t)^{2}}+\frac{2 \sqrt{(1+\mu)} \beta_{1} \Delta t}{(1-\alpha \Delta t)^{2}}+\widehat{K}_{16}(\Delta t)^{3 / 2}\right)\left\|X_{-k \tau+i \Delta t}^{-k \tau}-\widehat{X}_{-k \tau+i \Delta t}^{-k \tau}\right\|_{2}^{2} \\
& \quad+\widehat{K}_{15}(\Delta t)^{2}+\left(\frac{2 \sqrt{\widehat{K}_{7}}(\Delta t)^{3 / 2}}{1-\alpha \Delta t}+\widehat{K}_{17}(\Delta t)^{2}\right)\left\|X_{-k \tau+i \Delta t}^{-k \tau}-\widehat{X}_{-k \tau+i \Delta t}^{-k \tau}\right\|_{2} .
\end{aligned}
$$

Now we notice that the term $\left\|X_{-k \tau+i \Delta t}^{-k \tau}-\widehat{X}_{-k \tau+i \Delta t}^{-k \tau}\right\|_{2}^{2}$ has coefficients, the largest of which contains a constant multiplied by $\Delta t$. The largest free term contains a constant multiplied by $(\Delta t)^{2}$. Choosing $\mu$ and $\Delta t$ small enough and applying Young's inequality for the term $(\Delta t)^{3 / 2}\left\|X_{-k \tau+i \Delta t}^{-k \tau}-\widehat{X}_{-k \tau+i \Delta t}^{-k \tau}\right\|_{2}$, and from (4.2) we get

$$
\begin{aligned}
& (1-\alpha \Delta t)^{-2 M}\left\|X_{-k \tau+M \Delta t}^{-k \tau}-\widehat{X}_{-k \tau+M}^{-k \tau}\right\|_{2}^{2} \\
& \quad \leq \sum_{i=0}^{M-1}(1-\alpha \Delta t)^{-2 i}\left(\widehat{K}_{20} \Delta t\left\|X_{-k \tau+i \Delta t}^{-k \tau}-\widehat{X}_{-k \tau+i \Delta t}^{-k \tau}\right\|_{2}^{2}+\widehat{K}_{18}(\Delta t)^{2}\right) \\
& \quad \leq \widehat{K}_{19}(\Delta t)(1-\alpha \Delta t)^{-2 M}+\widehat{K}_{20}(\Delta t) \sum_{i=0}^{M-1}(1-\alpha \Delta t)^{-2 i}\left\|X_{-k \tau+i \Delta t}^{-k \tau}-\widehat{X}_{-k \tau+i \Delta t}^{-k \tau}\right\|_{2}^{2},
\end{aligned}
$$

where

$$
\widehat{K}_{19}=\frac{\widehat{K}_{18}(1-\alpha \Delta t)^{2}}{2 \alpha \Delta t-\alpha^{2}(\Delta t)^{2}}(\Delta t)=\frac{\widehat{K}_{18}(1-\alpha \Delta t)^{2}}{2 \alpha-\alpha^{2}(\Delta t)}, \widehat{K}_{20}=\frac{(1+\mu)\left(2 \beta_{1}+\beta_{2}^{2}+\varepsilon\right)}{(1-\alpha \Delta t)^{2}} .
$$

Here $\mu, \varepsilon$ and the time step $\Delta t$ are chosen small enough such that $\left(\widehat{K}_{20} \Delta t+1\right)(1-\alpha \Delta t)^{2}<1$. Now using the discrete time Gronwall inequality, from (4.10), we have

$$
\begin{aligned}
& \left\|X_{-k \tau+M \Delta t}^{-k \tau}-\widehat{X}_{-k \tau+M \Delta t}^{-k \tau}\right\|_{2}^{2} \\
& \quad \leq \widehat{K}_{19} \Delta t+\widehat{K}_{19} \widehat{K}_{20}(\Delta t)^{2} \frac{1-\left(\left(1+\widehat{K}_{20} \Delta t\right)(1-\alpha \Delta t)^{2}\right)^{M}}{1-\left(1+\widehat{K}_{20} \Delta t\right)(1-\alpha \Delta t)^{2}} \leq \widehat{K}_{21} \Delta t .
\end{aligned}
$$

We can find a constant $\widehat{K}_{21}$ which is independent of $M$ and $\Delta t$. Finally, we take $M=N+N^{\prime}$, where $N \Delta t=k \tau, N^{\prime} \in \mathbb{Z}$, then

$$
\begin{aligned}
\limsup _{k \rightarrow \infty}\left\|X_{N^{\prime} \Delta t}^{-k \tau}-\widehat{X}_{N^{\prime} \Delta t}^{-k \tau}\right\|_{2} & =\limsup _{N \rightarrow \infty}\left\|X_{-k \tau+\left(N+N^{\prime}\right) \Delta t}^{-k \tau}-\widehat{X}_{-k \tau+\left(N+N^{\prime}\right) \Delta t}^{-k \tau}\right\|_{2} \\
& \leq \sqrt{\widehat{K}_{21}} \sqrt{\Delta t}
\end{aligned}
$$

So we get the result.

We have proved the estimation of error from $-k \tau$ to $N^{\prime} \Delta t$ as $k \rightarrow \infty$ can be controlled under the $1 / 2$ order of the time step. And the upper bound is uniform in time. The following theorem will give us a more general result, which is from $-k \tau$ to time $r$. Let $\widehat{X}_{r}^{-k \tau}, r>0$ be given by (3.6). 
Theorem 4.2. Assume Conditions (A), (1) and (2). We choose $\Delta t=\tau / n$ for some $n \in \mathbb{N}, N=k n$. For any $r \geq 0$, there exists a constant $\widetilde{K}>0$ such that for any sufficiently small fixed $\Delta t$,

$$
\limsup _{k \rightarrow \infty}\left\|X_{r}^{-k \tau}-\widehat{X}_{r}^{-k \tau}\right\|_{2} \leq \widetilde{K} \sqrt{\Delta t}
$$

where $X_{r}^{-k \tau}$ is the exact solution, while $\widehat{X}_{r}^{-k \tau}$ is the numerical solution and $\widetilde{K}$ is independent of $\Delta t$ and $r$.

Proof. Assume for any $r \geq 0, N^{\prime}$ is the unique integer such that $N^{\prime} \Delta t \leq r,\left(N^{\prime}+1\right) \Delta t>r$. According to the semi-flow property, we have

$$
X_{r}^{-k \tau}(\omega)-\widehat{X}_{r}^{-k \tau}(\omega)=X_{r}^{N^{\prime} \Delta t}(\omega) \circ X_{N^{\prime} \Delta t}^{-k \tau}(\omega)-\widehat{X}_{r}^{N^{\prime} \Delta t}(\omega) \circ \widehat{X}_{N^{\prime} \Delta t}^{-k \tau}(\omega),
$$

where $\widehat{X}_{r}^{N^{\prime} \Delta t}$ is finite time Euler approximation of solution of (1.3) from $N^{\prime} \Delta t$ to $r$ and $\widehat{X}_{N^{\prime} \Delta t}^{-k \tau}$ is defined as before. So,

$$
\begin{aligned}
& \left\|X_{r}^{-k \tau}-\widehat{X}_{r}^{-k \tau}\right\|_{2} \\
& \quad \leq\left\|X_{r}^{N^{\prime} \Delta t} \circ X_{N^{\prime} \Delta t}^{-k \tau}-X_{r}^{N^{\prime} \Delta t} \circ \widehat{X}_{N^{\prime} \Delta t}^{-k \tau}\right\|_{2}+\left\|X_{r}^{N^{\prime} \Delta t} \circ \widehat{X}_{N^{\prime} \Delta t}^{-k \tau}-\widehat{X}_{r}^{N^{\prime} \Delta t} \circ \widehat{X}_{N^{\prime} \Delta t}^{-k \tau}\right\|_{2} .
\end{aligned}
$$

For the first term on the right-hand side, by Lemma 4.1, we have $\left\|X_{N^{\prime} \Delta t}^{-k \tau}-\widehat{X}_{N^{\prime} \Delta t}^{-k \tau}\right\| \leq K \sqrt{\Delta t}$. By the continuity of $X_{r}^{N^{\prime} \Delta t}(\cdot)$ with respect to initial values in $L^{2}(\Omega)$ [15], then

$$
\left\|X_{r}^{N^{\prime} \Delta t} \circ X_{N^{\prime} \Delta t}^{-k \tau}-X_{r}^{N^{\prime} \Delta t} \circ \widehat{X}_{N^{\prime} \Delta t}^{-k \tau}\right\|_{2} \leq C\left\|X_{N^{\prime} \Delta t}^{-k \tau}-\widehat{X}_{N^{\prime} \Delta t}^{-k \tau}\right\|_{2} \leq C_{5} \sqrt{\Delta t},
$$

where $C_{5}$ is independent of $\Delta t$. For the second term on the right-hand side of (4.11), it is finite time Euler approximation with same initial value. By Theorem 10.2.2 in Kloeden and Platen [14], there exists a constant $C_{6}>0$ such that for sufficiently $\Delta t>0$,

$$
\left\|X_{r}^{N^{\prime} \Delta t} \circ \widehat{X}_{N^{\prime} \Delta t}^{-k \tau}-\widehat{X}_{r}^{N^{\prime} \Delta t} \circ \widehat{X}_{N^{\prime} \Delta t}^{-k \tau}\right\|_{2} \leq C_{6} \sqrt{\Delta t},
$$

where the choice of $C_{6}$ is independent of $\Delta t$. The result follows by taking $\widetilde{K}=C_{5}+C_{6}$.

Corollary 4.3. For any $r \geq 0$, the exact and numerical approximating random periodic solutions of Eq. (1.3), $X_{r}^{*}$ and $\widehat{X}_{r}^{*}$, given in Theorems 2.4 and 3.4 , respectively, satisfy

$$
\left\|X_{r}^{*}-\widehat{X}_{r}^{*}\right\|_{2} \leq \widetilde{K} \sqrt{\Delta t}
$$

Proof. The result follows from

$$
\left\|X_{r}^{*}-\widehat{X}_{r}^{*}\right\|_{2} \leq \limsup _{k \rightarrow \infty}\left[\left\|X_{r}^{*}-X_{r}^{-k \tau}\right\|_{2}+\left\|X_{r}^{-k \tau}-\widehat{X}_{r}^{-k \tau}\right\|_{2}+\left\|\widehat{X}_{r}^{-k \tau}-\widehat{X}_{r}^{*}\right\|_{2}\right] .
$$

\subsection{Modified Milstein method}

For Milstein method, we can use the similar calculation as Euler-Maruyama scheme to get an improved error estimate between discrete approximate solution and the exact solution.

Theorem 4.4. Assume Conditions (A), (1') and (2). Then there exists a constant $K^{*}>0$ such that for any sufficiently small fixed $\Delta t$, the error between the exact solution $X_{r}^{-k \tau}$ and the numerical solution $\widehat{X}_{r}^{-k \tau}$ given by Milstein scheme (3.12) is $\lim \sup _{k \rightarrow \infty}\left\|X_{r}^{-k \tau}-\widehat{X}_{r}^{-k \tau}\right\|_{2} \leq K^{*} \Delta t$, for all $r \geq 0$, where $K^{*}$ is independent of $\Delta t$. 
Proof. In the following proof, we always denote by $\hat{K}$. the constant derived from the underlining computation unless otherwise stated. We consider the error in the similar way as Lemma 4.1.

$$
\begin{aligned}
& (1-\alpha \Delta t)^{-2 M}\left|X_{-k \tau+M \Delta t}^{-k \tau}-\widehat{X}_{-k \tau+M \Delta t}^{-k \tau}\right|^{2} \\
& \quad=\sum_{i=0}^{M-1}(1-\alpha \Delta t)^{-2 i}\left(\frac{\left|X_{-k \tau+(i+1) \Delta t}^{-k \tau}-\widehat{X}_{-k \tau+(i+1) \Delta t}^{-k \tau}\right|^{2}}{(1-\alpha \Delta t)^{2}}-\left|X_{-k \tau+i \Delta t}^{-k \tau}-\widehat{X}_{-k \tau+i \Delta t}^{-k \tau}\right|^{2}\right) .
\end{aligned}
$$

For simplicity we denote

$$
\begin{aligned}
\tilde{B}_{1}= & \frac{1}{1-\alpha \Delta t} \int_{i \Delta t-k \tau}^{(i+1) \Delta t-k \tau}\left[e^{-A(s+k \tau-(i+1) \Delta t)} f\left(s, X_{s}^{-k \tau}\right)-f\left(i \Delta t, \widehat{X}_{-k \tau+i \Delta t}^{-k \tau}\right)\right. \\
& \left.-\int_{i \Delta t-k \tau}^{s} F_{i}^{(1)}\left(\widehat{X}_{-k \tau+i \Delta t}^{-k \tau}\right) d W_{v}\right] d s . \\
\tilde{B}_{2}= & \frac{1}{1-\alpha \Delta t} \int_{i \Delta t-k \tau}^{(i+1) \Delta t-k \tau}\left[e^{-A(s+k \tau-(i+1) \Delta t)} g\left(s, X_{s}^{-k \tau}\right)-g\left(i \Delta t, \widehat{X}_{-k \tau+i \Delta t}^{-k \tau}\right)\right. \\
& \left.-\int_{i \Delta t-k \tau}^{s} G_{i}^{(1)}\left(\widehat{X}_{-k \tau+i \Delta t}^{-k \tau}\right) d W_{v}\right] d W_{s},
\end{aligned}
$$

with

$$
\begin{aligned}
F_{i}^{(1)}(x) & =\frac{1}{2 \sqrt{\Delta t}}\left(f\left(i \Delta t, \widehat{\Upsilon}_{+}(x)\right)-f\left(i \Delta t, \widehat{\Upsilon}_{-}(x)\right)\right), \\
G_{i}^{(1)}(x) & =\frac{1}{2 \sqrt{\Delta t}}\left(g\left(i \Delta t, \widehat{\Upsilon}_{+}(x)\right)-g\left(i \Delta t, \widehat{\Upsilon}_{-}(x)\right)\right) .
\end{aligned}
$$

Therefore,

$$
\begin{aligned}
& X_{-k \tau+(i+1) \Delta t}^{-k \tau}-\widehat{X}_{-k \tau+(i+1) \Delta t}^{-k \tau} \\
& \quad=e^{A \Delta t} X_{-k \tau+i \Delta t}^{-k \tau}-(I+A \Delta t) \widehat{X}_{-k \tau+i \Delta t}^{-k \tau}+(1-\alpha \Delta t)\left(\tilde{B}_{1}+\tilde{B}_{2}\right) .
\end{aligned}
$$

Now we consider

$$
\begin{aligned}
& \frac{\left|X_{-k \tau+(i+1) \Delta t}^{-k \tau}-\widehat{X}_{-k \tau+(i+1) \Delta t}^{-k \tau}\right|^{2}}{(1-\alpha \Delta t)^{2}}-\left|X_{-k \tau+i \Delta t}^{-k \tau}-\widehat{X}_{-k \tau+i \Delta t}^{-k \tau}\right|^{2} \\
& =\left(X_{-k \tau+i \Delta t}^{-k \tau}-\widehat{X}_{-k \tau+i \Delta t}^{-k \tau}\right)^{T}\left(\frac{e^{A \Delta t}}{1-\alpha \Delta t}-I\right)\left(\frac{e^{A \Delta t}}{1-\alpha \Delta t}+I\right) \\
& \quad \times\left(X_{-k \tau+i \Delta t}^{-k \tau}-\widehat{X}_{-k \tau+i \Delta t}^{-k \tau}\right) \\
& \quad+\left(\widehat{X}_{-k \tau+i \Delta t}^{-k \tau}\right)^{T}\left(\frac{e^{A \Delta t}-I-A \Delta t}{1-\alpha \Delta t}\right)^{2}\left(\widehat{X}_{-k \tau+i \Delta t}^{-k \tau}\right)+\tilde{B}_{1}^{T} \tilde{B}_{1}+\tilde{B}_{2}^{T} \tilde{B}_{2} \\
& \quad+2\left(X_{-k \tau+i \Delta t}^{-k \tau}-\widehat{X}_{-k \tau+i \Delta t}^{-k \tau}\right)^{T}\left(\frac{e^{A \Delta t}}{1-\alpha \Delta t}\right)\left(\frac{e^{A \Delta t}-I-A \Delta t}{1-\alpha \Delta t}\right)\left(\widehat{X}_{-k \tau+i \Delta t}^{-k \tau}\right)
\end{aligned}
$$




$$
\begin{aligned}
& +2\left(\left(X_{-k \tau+i \Delta t}^{-k \tau}\right)^{T}\left(\frac{e^{A \Delta t}}{1-\alpha \Delta t}\right)-\left(\widehat{X}_{-k \tau+i \Delta t}^{-k \tau}\right)^{T}\left(\frac{I+A \Delta t}{1-\alpha \Delta t}\right)\right) \tilde{B}_{1} \\
& +2\left(\left(X_{-k \tau+i \Delta t}^{-k \tau}\right)^{T}\left(\frac{e^{A \Delta t}}{1-\alpha \Delta t}\right)-\left(\widehat{X}_{-k \tau+i \Delta t}^{-k \tau}\right)^{T}\left(\frac{I+A \Delta t}{1-\alpha \Delta t}\right)\right) \tilde{B}_{2}+2 \tilde{B}_{1}^{T} \tilde{B}_{2} .
\end{aligned}
$$

By the similar analysis as (4.5) and (4.7), we have

$$
\begin{aligned}
\mathbb{E}\left[\tilde{B}_{1}^{T} \tilde{B}_{1}\right] \leq & \widehat{K}_{22}(\Delta t)^{4}+\frac{(1+\mu) \beta_{1}^{2}(\Delta t)^{2}}{(1-\alpha \Delta t)^{2}}\left\|X_{-k \tau+i \Delta t}^{-k \tau}-\widehat{X}_{-k \tau+i \Delta t}^{-k \tau}\right\|_{2}^{2} \\
& +\widehat{K}_{23}^{2}(\Delta t)^{3}\left\|X_{-k \tau+i \Delta t}^{-k \tau}-\widehat{X}_{-k \tau+i \Delta t}^{-k \tau}\right\|_{2}^{2}
\end{aligned}
$$

and

$$
\begin{aligned}
\mathbb{E}\left[\tilde{B}_{2}^{T} \tilde{B}_{2}\right] \leq & \widehat{K}_{24}(\Delta t)^{3}+\frac{(1+\mu) \beta_{2}^{2} \Delta t}{(1-\alpha \Delta t)^{2}}\left\|X_{-k \tau+i \Delta t}^{-k \tau}-\widehat{X}_{-k \tau+i \Delta t}^{-k \tau}\right\|_{2}^{2} \\
& +\widehat{K}_{25}(\Delta t)^{2}\left\|X_{-k \tau+i \Delta t}^{-k \tau}-\widehat{X}_{-k \tau+i \Delta t}^{-k \tau}\right\|_{2}^{2} .
\end{aligned}
$$

The crossing product terms in (4.13) are estimated similar as (4.8) as follows:

$$
\begin{aligned}
\mathbb{E}\left[2\left(\left(X_{-k \tau+i \Delta t}^{-k \tau}\right)^{T}\left(\frac{e^{A \Delta t}}{1-\alpha \Delta t}\right)-\left(\widehat{X}_{-k \tau+i \Delta t}^{-k \tau}\right)^{T}\left(\frac{I+A \Delta t}{1-\alpha \Delta t}\right)\right) \tilde{B}_{1}\right] \\
\leq \widehat{K}_{26}(\Delta t)^{4}+\frac{\sqrt{1+\mu} \beta_{1} \widehat{K}_{27}(\Delta t)^{3}}{(1-\alpha \Delta t)^{2}}\left\|X_{-k \tau+i \Delta t}^{-k \tau}-\widehat{X}_{-k \tau+i \Delta t}^{-k \tau}\right\|_{2} \\
\quad+\frac{2 \sqrt{\widehat{K}_{22}}(\Delta t)^{2}}{1-\alpha \Delta t}\left\|X_{-k \tau+i \Delta t}^{-k \tau}-\widehat{X}_{-k \tau+i \Delta t}^{-k \tau}\right\|_{2}(1+\Delta t|A|) \\
\quad+\frac{2 \sqrt{1+\mu} \beta_{1} \Delta t}{(1-\alpha \Delta t)^{2}}\left\|X_{-k \tau+i \Delta t}^{-k \tau}-\widehat{X}_{-k \tau+i \Delta t}^{-k \tau}\right\|_{2}^{2}(1+\Delta t|A|) \\
+2 \widehat{K}_{23}(\Delta t)^{3 / 2}\left\|X_{-k \tau+i \Delta t}^{-k \tau}-\widehat{X}_{-k \tau+i \Delta t}^{-k \tau}\right\|_{2}^{2}(1+\Delta t|A|) .
\end{aligned}
$$

The seventh term remains 0 under conditional expectation.

$$
\mathbb{E}\left[\left(\left(X_{-k \tau+i \Delta t}^{-k \tau}\right)^{T}\left(\frac{e^{A \Delta t}}{1-\alpha \Delta t}\right)-\left(\widehat{X}_{-k \tau+i \Delta t}^{-k \tau}\right)^{T}\left(\frac{I+A \Delta t}{1-\alpha \Delta t}\right)\right) \tilde{B}_{2}\right]=0 .
$$

For the last term,

$$
\begin{aligned}
\mathbb{E}\left[2 \tilde{B}_{1}^{T} \tilde{B}_{2}\right] & \leq 2\left\|\tilde{B}_{1}^{T}\right\|_{2} \cdot\left\|\tilde{B}_{2}\right\|_{2} \\
& \leq \widehat{K}_{28}(\Delta t)^{7 / 2}+\widehat{K}_{29}(\Delta t)^{3 / 2}\left\|X_{-k \tau+i \Delta t}^{-k \tau}-\widehat{X}_{-k \tau+i \Delta t}^{-k \tau}\right\|_{2}^{2} .
\end{aligned}
$$

Combining all the estimation above, we have

$$
\begin{aligned}
& \frac{\left|X_{-k \tau+(i+1) \Delta t}^{-k \tau}-\widehat{X}_{-k \tau+(i+1) \Delta t}^{-k \tau}\right|^{2}}{(1-\alpha \Delta t)^{2}}-\left|X_{-k \tau+i \Delta t}^{-k \tau}-\widehat{X}_{-k \tau+i \Delta t}^{-k \tau}\right|^{2} \\
& \leq\left(\frac{(1+\mu) \beta_{2}^{2} \Delta t}{(1-\alpha \Delta t)^{2}}+\frac{2 \sqrt{(1+\mu)} \beta_{1} \Delta t}{(1-\alpha \Delta t)^{2}}+\widehat{K}_{40}(\Delta t)^{3 / 2}\right)\left\|X_{-k \tau+i \Delta t}^{-k \tau}-\widehat{X}_{-k \tau+i \Delta t}^{-k \tau}\right\|_{2}^{2} \\
& \quad+\widehat{K}_{41}(\Delta t)^{3}+\widehat{K}_{42}(\Delta t)^{2}\left\|X_{-k \tau+i \Delta t}^{-k \tau}-\widehat{X}_{-k \tau+i \Delta t}^{-k \tau}\right\|_{2} .
\end{aligned}
$$


Choosing $\mu$ and $\Delta t$ small enough and applying Young's inequality to the term $(\Delta t)^{2}\left\|X_{-k \tau+i \Delta t}^{-k \tau}-\widehat{X}_{-k \tau+i \Delta t}^{-k \tau}\right\|_{2}$, and from (4.12) we get

$$
\begin{aligned}
& (1-\alpha \Delta t)^{-2 M}\left\|X_{-k \tau+M \Delta t}^{-k \tau}-\widehat{X}_{-k \tau+M \Delta t}^{-k \tau}\right\|_{2}^{2} \\
& \quad \leq \sum_{i=0}^{M-1}(1-\alpha \Delta t)^{-2 i}\left(\widehat{K}_{43} \Delta t\left\|X_{-k \tau+i \Delta t}^{-k \tau}-\widehat{X}_{-k \tau+i \Delta t}^{-k \tau}\right\|_{2}^{2}+\widehat{K}_{44}(\Delta t)^{3}\right) \\
& \quad \leq \widehat{K}_{45}(\Delta t)^{2}(1-\alpha \Delta t)^{-2 M}+\widehat{K}_{43}(\Delta t) \sum_{i=0}^{M-1}(1-\alpha \Delta t)^{-2 i}\left\|X_{-k \tau+i \Delta t}^{-k \tau}-\widehat{X}_{-k \tau+i \Delta t}^{-k \tau}\right\|_{2}^{2},
\end{aligned}
$$

where

$$
\widehat{K}_{45}=\frac{\widehat{K}_{44}(1-\alpha \Delta t)^{2}}{2 \alpha \Delta t-\alpha^{2}(\Delta t)^{2}}(\Delta t)=\frac{\widehat{K}_{44}(1-\alpha \Delta t)^{2}}{2 \alpha-\alpha^{2}(\Delta t)}, \widehat{K}_{43}=\frac{(1+\mu)\left(2 \beta_{1}+\beta_{2}^{2}+\varepsilon\right)}{(1-\alpha \Delta t)^{2}}
$$

Here $\mu, \varepsilon$ and the time step $\Delta t$ are chosen small enough such that $\left(\widehat{K}_{43} \Delta t+1\right)(1-\alpha \Delta t)^{2}<1$. Applying the discrete time Gronwall inequality, from (4.16), we have

$$
\begin{aligned}
& \left\|X_{-k \tau+M \Delta t}^{-k \tau}-\widehat{X}_{-k \tau+M \Delta t}^{-k \tau}\right\|_{2}^{2} \\
& \quad \leq \widehat{K}_{45}(\Delta t)^{2}+\widehat{K}_{45} \widehat{K}_{43}(\Delta t)^{2} \frac{1-\left(\left(1+\widehat{K}_{43} \Delta t\right)(1-\alpha \Delta t)^{2}\right)^{M}}{1-\left(1+\widehat{K}_{43} \Delta t\right)(1-\alpha \Delta t)^{2}} \leq \widehat{K}_{46}(\Delta t)^{2} .
\end{aligned}
$$

We can find a constant $\widehat{K}_{46}$ which is independent of $M$ and $\Delta t$. We take $M=N$, where $N \Delta t=k \tau$, then

$$
\limsup _{k \rightarrow \infty}\left\|X_{0}^{-k \tau}-\widehat{X}_{0}^{-k \tau}\right\|_{2}=\limsup _{N \rightarrow \infty}\left\|X_{-k \tau+N \Delta t}^{-k \tau}-\widehat{X}_{-k \tau+N \Delta t}^{-k \tau}\right\|_{2} \leq \sqrt{\widehat{K}_{46}} \Delta t .
$$

The discussion about the convergence from time $-k \tau$ to $r$ is the same as Theorem 4.2 as we know that the Milstein scheme with addition term also has strong order 1.0 for finite horizon.

Remark 4.5. Compared with Euler-Maruyama scheme, the order 1.0 Milstein method improved the order by replacing terms $B_{1}$ and $B_{2}$ with more accurate approximation $\tilde{B}_{1}$ and $\tilde{B}_{2}$. If we did not have the additional term

$$
\left.\frac{\Delta Z_{i}}{2 \sqrt{\Delta t}}\left[f\left(i \Delta t, \widehat{\Upsilon}_{+}\left(\widehat{X}_{-k \tau+i \Delta t}^{-k \tau}\right)\right)\right)-f\left(i \Delta t, \widehat{\Upsilon}_{-}\left(\widehat{X}_{-k \tau+i \Delta t}^{-k \tau}\right)\right)\right],
$$

we would only have the result with $B_{1}$ and $\tilde{B}_{2}$.

Here if we compare the scheme without additional term, it is important to notice that the term $\left\|X_{-k \tau+i \Delta t}^{-k \tau}-\widehat{X}_{-k \tau+i \Delta t}^{-k \tau}\right\|_{2}$ in (4.14) is multiplied by $(\Delta t)^{2}$. But in (4.9) it is multiplied by $(\Delta t)^{3 / 2}$. When we apply the Young's inequality in (4.15), to make sure the free term with $(\Delta t)^{3}$, we have

$$
\widehat{K}_{42}(\Delta t)^{\frac{3}{2}}\left\|X_{-k \tau+i \Delta t}^{-k \tau}-\widehat{X}_{-k \tau+i \Delta t}^{-k \tau}\right\|_{2} \leq \widehat{K}_{47}(\Delta t)^{3}+\widehat{K}_{48}\left\|X_{-k \tau+i \Delta t}^{-k \tau}-\widehat{X}_{-k \tau+i \Delta t}^{-k \tau}\right\|_{2}^{2} .
$$

This will influence the constant $\widehat{K}_{43}$ in (4.16) to fail the inequality $\left(\widehat{K}_{43} \Delta t+1\right)(1-\alpha \Delta t)^{2}<1$. On the finite horizon, $\widehat{K}_{46}$ is still bounded by the boundedness of $M$. But in the case of the infinite horizon, the scheme is under the risk of instability. For this reason, we modify the scheme with the additional term from higher-order scheme. 

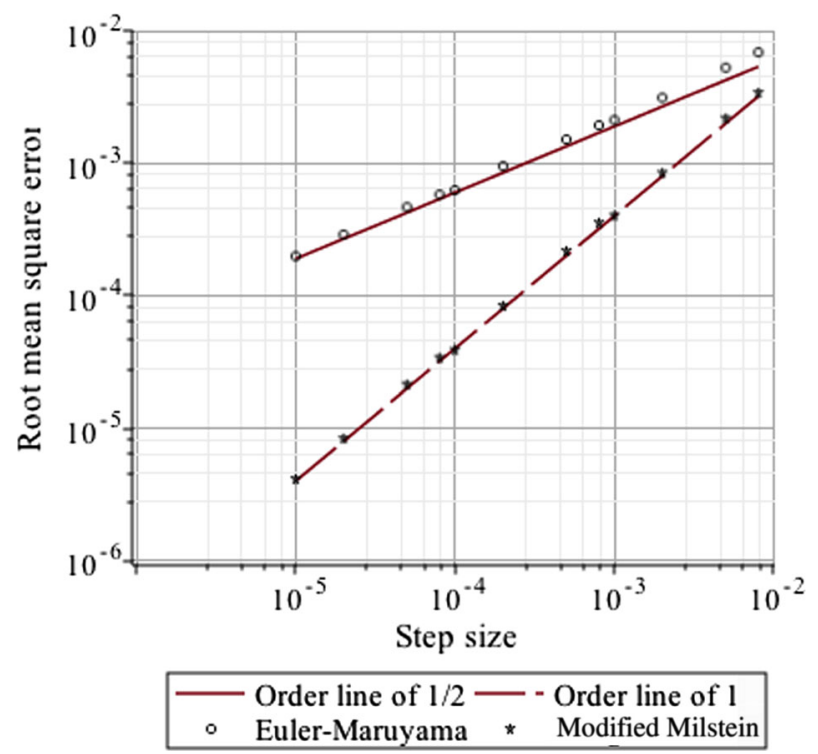

FIG. 3. Root-mean-square error versus step size as log-log plot for the SDE (3.11)

Corollary 4.6. For any $r \geq 0$, the exact and numerical approximating random periodic solutions of Eq. (1.3), $X_{r}^{*}$ and $\widehat{X}_{r}^{*}$, given in Theorems 2.4 and 3.6 , respectively, satisfy

$$
\left\|X_{r}^{*}-\widehat{X}_{r}^{*}\right\|_{2} \leq K^{*} \Delta t
$$

Here $K^{*}$ is independent of $\Delta t$ and $r$.

Example 4.7. To illustrate the errors in Theorems 4.2 and 4.4, we simulate the random periodic solution of Example 1 with 2000 different noise realisations by both Euler-Maruyama method and modified Milstein method. We then apply Monte Carlo method to obtain the root-mean-square errors between the exact random periodic solution and the respective numerical schemes with 12 different step sizes: $1 \times 10^{-5}, 2 \times 10^{-5}, 3 \times 10^{-5}, 4 \times 10^{-5}, 1 \times 10^{-4}, 2 \times 10^{-4}, 3 \times 10^{-4}, 4 \times 10^{-4}, 1 \times 10^{-3}, 2 \times 10^{-3}, 3 \times 10^{-3}, 4 \times 10^{-3}$, where the exact one is given explicitly as $X_{t}^{*}=\int_{-\infty}^{t} \mathrm{e}^{-\left(\pi+\frac{1}{2}\right)(t-s)+W_{t}-W_{s}} \sin (\pi s) d s$. The relationship between the root-mean-square errors and the step size is shown in the log-log plot in Fig. 3. The difference of the orders of convergence between the Euler-Maruyama method and Milstein method is clear from the numerical simulations.

\section{Periodic measures}

Let $\mathcal{P}\left(\mathbb{R}^{m}\right)$ denote all probability measures on $\mathbb{R}^{m}$. For $P_{1}, P_{2} \in \mathcal{P}\left(\mathbb{R}^{m}\right)$, define metric $d_{\mathbb{L}}$ as follows:

$$
d_{\mathbb{L}}\left(P_{1}, P_{2}\right)=\sup _{\varphi \in \mathbb{L}}\left|\int_{\mathbb{R}^{m}} \varphi(x) P_{1}(d x)-\int_{\mathbb{R}^{m}} \varphi(x) P_{2}(d x)\right|,
$$

where

$$
\mathbb{L}=\left\{\varphi: \mathbb{R}^{m} \rightarrow \mathbb{R}:|\varphi(x)-\varphi(y)| \leq|x-y| \text { and }|\varphi(\cdot)| \leq 1\right\}
$$


From [11], it is not difficult to prove that the metric $d_{\mathbb{L}}$ is equivalent to the weak topology. This useful observation was made in [27].

We can define the transition probability of the semi-flow $u$ which is generated by the solution of (1.4) as follows:

$$
P(t+s, s, \xi, \Gamma):=P(\{\omega: u(t+s, s, \omega) \xi \in \Gamma\})=P\left(X_{t+s}^{s}(\xi) \in \Gamma\right),
$$

for any $\Gamma \in \mathcal{B}\left(\mathbb{R}^{m}\right)$. For any $\varphi$ being bounded and measurable

$$
P(t+s, s) \varphi(\xi)=\int_{\mathbb{R}^{m}} P(t+s, s, \xi, d \eta) \varphi(\eta)=E \varphi\left(X_{t+s}^{s}(\xi)\right)
$$

defines a semi-group satisfying

$$
P(t+s+r, s+r) \circ P(s+r, s)=P(t+s+r, s), r, t \geq 0, s \in \mathbb{R} .
$$

Recall the following definition of periodic measure given in [8] .

Definition 5.1. [8] The measure function $\rho .: \mathbb{R} \rightarrow \mathcal{P}\left(\mathbb{R}^{m}\right)$ is called periodic measure if it satisfies for any $s \in \mathbb{R}, t \geq 0$, and $\Gamma \in \mathcal{B}\left(\mathbb{R}^{m}\right)$,

$$
\rho_{s+\tau}=\rho_{s}, \int_{\mathbb{R}^{m}} P(t+s, s, x, \Gamma) \rho_{s}(d x)=\rho_{t+s}(\Gamma) .
$$

From Theorem 2.4, we know that the random periodic solution of (1.4) exists. So by the result in [8], we know that the periodic measure $\rho$. exists, which can be defined as the law of random periodic solutions, i.e.

$$
\rho_{r}(\Gamma)=P\left(X_{r}^{*} \in \Gamma\right) .
$$

Similarly, we can define the transition probability of the discrete semi-flow $\hat{u}$ from Euler-Maruyama scheme by

$$
\hat{P}(t+s, s, \xi, \Gamma):=P(\{\omega: \hat{u}(t+s, s, \omega) \xi \in \Gamma\})=P\left(\hat{X}_{t+s}^{s}(\xi) \in \Gamma\right) .
$$

For any $\varphi$ being bounded and measurable

$$
\hat{P}(t+s, s) \varphi(\xi)=\int_{\mathbb{R}^{m}} \hat{P}(t+s, s, \xi, d \eta) \varphi(\eta)=E \varphi\left(\hat{X}_{t+s}^{s}(\xi)\right)
$$

defines a semi-group satisfying

$$
\hat{P}(t+s+r, s+r) \circ \hat{P}(s+r, s)=\hat{P}(t+s+r, s), r, t \geq 0, s \in \mathbb{R},
$$

Similar to the result in [8], the measure function defined by

$$
\hat{\rho}_{r}(\Gamma)=P\left(\hat{X}_{r}^{*} \in \Gamma\right)
$$

is a periodic measure for Markov semi-group $\hat{P}(t+s, s)$. It satisfies for any $s \in \mathbb{R}, t \geq 0$, and $\Gamma \in \mathcal{B}\left(\mathbb{R}^{m}\right)$,

$$
\hat{\rho}_{s+\tau}=\hat{\rho}_{s}, \int_{\mathbb{R}^{m}} \hat{P}(t+s, s, x, \Gamma) \hat{\rho}_{s}(d x)=\hat{\rho}_{t+s}(\Gamma) .
$$

We have following error estimate of $\rho$. and $\hat{\rho}$. Consider the Euler-Maruyama scheme (3.1) first.

Theorem 5.2. Assume Conditions (A), (1) and (2). Then periodic measures $\rho$. and $\hat{\rho}$. of the Markov semi-group generated by the exact solution of (1.3) and approximation (3.1) are weak limits of transition probabilities along integral multiples of period, i.e.

$$
P(r,-k \tau, \xi) \rightarrow \rho_{r}, \hat{P}(r,-k \tau, \xi) \rightarrow \hat{\rho}_{r}, \text { as } \mathrm{k} \rightarrow \infty,
$$


weakly and the error estimate is

$$
d_{\mathbb{L}}\left(\rho_{r}, \widehat{\rho}_{r}\right) \leq \widetilde{K} \sqrt{\Delta t}
$$

where $\widetilde{K}$ is independent of $\Delta t$ and $r$.

Proof. To prove (5.5), by (5.1), (5.2), Theorem 2.4 and Jensen's inequality, we have

$$
\begin{aligned}
d_{\mathbb{L}} & \left(P(r,-k \tau, \xi), \rho_{r}\right) \\
& =\sup _{\varphi \in \mathbb{L}}\left|\int_{\mathbb{R}^{m}} \varphi(x) P(r,-k \tau, \xi, d x)-\int_{\mathbb{R}^{m}} \varphi(x) \rho_{r}(d x)\right| \\
& =\sup _{\varphi \in \mathbb{L}}\left|\mathbb{E}\left[\varphi\left(X_{r}^{-k \tau}\right)-\varphi\left(X_{r}^{*}\right)\right]\right| \\
& \leq \sup _{\varphi \in \mathbb{L}} \mathbb{E}\left|\varphi\left(X_{r}^{-k \tau}\right)-\varphi\left(X_{r}^{*}\right)\right| \\
& \leq \mathbb{E}\left|X_{r}^{-k \tau}-X_{r}^{*}\right| \\
& \leq\left\|X_{r}^{-k \tau}-X_{r}^{*}\right\|_{2} \\
& \rightarrow 0
\end{aligned}
$$

as $k \rightarrow \infty$. So $P(r,-k \tau, \xi) \rightarrow \rho_{r}$ weakly as $k \rightarrow \infty$ from the well-known result in [11]. Similarly, we can have for the discrete system, $\hat{P}(r,-k \tau, \xi) \rightarrow \hat{\rho}_{r}$ weakly as $k \rightarrow \infty$. Now we consider the metric between these two periodic measures $\rho$. and $\hat{\rho}$.,

$$
\begin{aligned}
d_{\mathbb{L}}\left(\rho_{r}, \widehat{\rho}_{r}\right)= & \sup _{\varphi \in \mathbb{L}}\left|\int_{\mathbb{R}^{m}} \varphi(x) \rho_{r}(d x)-\int_{\mathbb{R}^{m}} \varphi(x) \widehat{\rho}_{r}(d x)\right| \\
\leq & \sup _{\varphi \in \mathbb{L}}\left|\int_{\mathbb{R}^{m}} \varphi(x) \rho_{r}(d x)-\int_{\mathbb{R}^{m}} \varphi(x) P(r,-k \tau, \xi, d x)\right| \\
& +\sup _{\varphi \in \mathbb{L}}\left|\int_{\mathbb{R}^{m}} \varphi(x) P(r,-k \tau, \xi, d x)-\int_{\mathbb{R}^{m}} \varphi(x) \widehat{P}(r,-k \tau, \xi, d x)\right| \\
& +\sup _{\varphi \in \mathbb{L}}\left|\int_{\mathbb{R}^{m}} \varphi(x) \widehat{P}(r,-k \tau, \xi, d x)-\int_{\mathbb{R}^{m}} \varphi(x) \widehat{\rho}_{r}(d x)\right| \\
= & \sup _{\varphi \in \mathbb{L}}\left|\mathbb{E}\left[\varphi\left(X_{r}^{*}\right)-\varphi\left(X_{r}^{-k \tau}\right)\right]\right|+\sup _{\varphi \in \mathbb{L}}\left|\mathbb{E}\left[\varphi\left(X_{r}^{-k \tau}\right)-\varphi\left(\widehat{X}_{r}^{-k \tau}\right)\right]\right| \\
& +\sup _{\varphi \in \mathbb{L}}\left|\mathbb{E}\left[\varphi\left(\widehat{X}_{r}^{-k \tau}\right)-\varphi\left(\widehat{X}_{r}^{*}\right)\right]\right| \\
\leq & \mathbb{E}\left|X_{r}^{*}-X_{r}^{-k \tau}\right|+\mathbb{E}\left|X_{r}^{-k \tau}-\widehat{X}_{r}^{-k \tau}\right|+\mathbb{E}\left|\widehat{X}_{r}^{-k \tau}-\widehat{X}_{r}^{*}\right| \\
\leq & \left.\left\|X_{r}^{*}-X_{r}^{-k \tau}\right\|\right|_{2}+\left.\left\|X_{r}^{-k \tau}-\widehat{X}_{r}^{-k \tau}\right\|\right|_{2}+\left\|\hat{X}_{r}^{-k \tau}-X_{r}^{*}\right\|_{2} .
\end{aligned}
$$

By Theorems 2.4, 3.4, 4.2, we have for any $\epsilon>0$, there exists $N>0$ such that when $k \geq N$,

and

$$
\left\|X_{r}^{*}-X_{r}^{-k \tau}\right\|_{2} \leq \frac{\epsilon}{3},\left\|\hat{X}_{r}^{-k \tau}-X_{r}^{*}\right\|_{2} \leq \frac{\epsilon}{3}
$$

$$
\left\|X_{r}^{-k \tau}-\widehat{X}_{r}^{-k \tau}\right\|_{2} \leq \widetilde{K} \sqrt{\Delta t}+\frac{\epsilon}{3}
$$


Then taking $k \geq N$ in (5.7), we have

$$
d_{\mathbb{L}}\left(\rho_{r}, \widehat{\rho}_{r}\right) \leq \widetilde{K} \sqrt{\Delta t}+\epsilon
$$

Note in the above inequality, the left-hand side does not depend on $k$ and $\epsilon$ is arbitrary. So (5.6) is obtained.

Remark 5.3. There are a number of works about approximating of invariant measures for SDE using Euler-Maruyama method and Milstein method [16,22,23,27]. For finite horizon, the order of weak convergence of Euler-Maruyama method was proved to be 1.0, a significant improvement from the order 0.5 in the strong convergence (c.f. [14]). However, the order of 1.0 is not guaranteed in the infinite horizon case, see [16] for the case of the invariant measures. On the other hand, in some work such as [22,23], the order of error of Euler-Maruyama method was managed to increase to 1.0 under the non-degenerate condition. Here we do not have such an assumption, and we have order 0.5 in the weak convergence formulation. However, in the case of the modified Milstein method, we will see that the error is of order 1.0 in the next theorem. Note that the error estimate with the Milstein scheme is also 1.0 in the weak convergence formulation even in the non-degenerate case [22,23].

Theorem 5.4. Assume Conditions (A), (1') and (2). Consider the modified Milstein scheme (3.12). Then the periodic measure $\hat{\rho}$. of the Markov semi-groups generated by the discretised semi-flow is the weak limit of its transition probability along integral multiples of period, i.e.

$$
\hat{P}(r,-k \tau, \xi) \rightarrow \hat{\rho}_{r}, \text { as } \mathrm{k} \rightarrow \infty,
$$

weakly and the error estimate between the approximating periodic measure $\hat{\rho}$. and the exact periodic measure is

$$
d_{\mathbb{L}}\left(\rho_{r}, \widehat{\rho}_{r}\right) \leq K^{*} \Delta t
$$

where $K^{*}$ is independent of $\Delta t$ and $r$.

Proof. The proof is similar to the proof of Theorem 5.2, but using Theorem 4.4 instead of Theorem 4.2.

\section{Transformation of the periodic SDE via Lyapunov-Floquet transformation}

In this section, we consider the following m-dimensional system

$$
d X_{t}^{t_{0}}=A(t) X_{t}^{t_{0}} d t+\widetilde{f}\left(t, X_{t}^{t_{0}}\right) d t+\widetilde{g}\left(t, X_{t}^{t_{0}}\right) d W_{t}, t \geq t_{0},
$$

with $X_{t_{0}}^{t_{0}}=\xi$. We assume that the matrix $A(t)$ is a continuous $\tau$-periodic $m \times m$ real matrix and the functions $\tilde{f}$ and $\widetilde{g}$ are both $\tau$-periodic in time, i.e.

$$
A(t+\tau)=A(t), \widetilde{f}(t+\tau, \cdot)=\widetilde{f}(t, \cdot), \widetilde{g}(t+\tau, \cdot)=\widetilde{g}(t, \cdot), \text { for any } t \in \mathbb{R} .
$$

To solve this problem we need to apply the Floquet theorem to transfer this system to a system with the linear part having a time-invariant generator.

\subsection{The transformation}

The well-known Floquet theorem can be found in many books, such as [9]. It says that if $\Phi(t)$ is a fundamental matrix solution of the periodic system $\dot{X}=A(t) X$, then so is $\Phi(t+\tau)$. Moreover, there exists an invertible $\tau$-periodic matrix $P(t)$ such that $\Phi(t)=P(t) e^{R t}$, where $R$ is a constant matrix. The matrix $P(t)$ is called the Lyapunov-Floquet transformation matrix, and $X=P(t) Z$ is called the Lyapunov-Floquet transformation. 
Proposition 6.1. Under Lyapunov-Floquet transformation $X(t)=P(t) Z(t)$, periodic system (6.1) is transferred to the following system with constant coefficient matrix linear part

$$
d Z_{t}^{t_{0}}=R Z_{t}^{t_{0}} d t+P(t)^{-1} \tilde{f}\left(t, P(t) Z_{t}^{t_{0}}\right) d t+P(t)^{-1} \widetilde{g}\left(t, P(t) Z_{t}^{t_{0}}\right) d W_{t},
$$

with $Z_{t_{0}}^{t_{0}}=P\left(t_{0}\right)^{-1} \xi$.

Proof. The proof follows some elementary calculations.

From the periodicity of $P$, we know that

$$
\Phi(t+\tau)=P(t+\tau) e^{R(t+\tau)}=P(t) e^{R t} e^{R \tau}=\Phi(t) e^{R \tau} .
$$

Since $e^{R+2 \pi k i I}=e^{R} e^{2 \pi k i} I=e^{R}$ for any $k \in \mathbb{Z}$, the constant matrix $R$ is not unique. It is also not necessarily real, even if $e^{R \tau}$ is real. So we need the following corollary to guarantee such a real constant matrix exists.

Corollary 6.2. Let $B=\frac{R+\bar{R}}{2}, S(t)=\Phi(t) e^{-B t}$. Then $S(t)$ is real and $2 \tau$-periodic. Under the transformation $X_{t}^{t_{0}}=S(t) Z_{t}^{t_{0}}$, periodic system (6.1) is transferred to the following system with constant coefficient matrix linear part

$$
d Z_{t}^{t_{0}}=B Z_{t}^{t_{0}} d t+S(t)^{-1} \widetilde{f}\left(t, S(t) Z_{t}^{t_{0}}\right) d t+S(t)^{-1} \widetilde{g}\left(t, S(t) Z_{t}^{t_{0}}\right) d W_{t},
$$

with $Z_{t_{0}}^{t_{0}}=S\left(t_{0}\right)^{-1} \xi$,

Proof. Because $A(t)$ is real, so the matrix $C=e^{R \tau}=\Phi(\tau) \Phi^{-1}(0)$ is real. Thus, for the real matrix $B=\frac{R+\bar{R}}{2}, C^{2}=e^{R \tau} e^{\bar{R} \tau}=e^{2 B \tau}$. Note $S(t)$ is real since $B$ is real. And notice that

$$
S(t+2 \tau)=\Phi(t+2 \tau) e^{-B(t+2 \tau)}=\Phi(t) C^{2} e^{-2 B \tau} e^{-B t}=\Phi(t) e^{-B t}=S(t) .
$$

Then we can obtain the time-invariant system in a similar way as in the Corollary 6.1. The only difference is that the system with real constant coefficient matrix linear part becomes $2 \tau$-periodic.

\subsection{Convergence theorem of the periodic parameter matrix system}

Condition $\left(\mathbf{A}^{\prime}\right)$. The matrix function $A(t)$ is $\tau$-periodic, the corresponding matrix $B$ is symmetric with eigenvalues satisfying $0>\lambda_{1} \geq \lambda_{2} \geq \ldots \geq \lambda_{m}$.

Because $S(t)$ is continuous and periodic, so $S(t)$ is bounded. The periodicity and continuity of $S(t)^{-1}$ are obtained from the properties of $S(t)$, and it is concluded that $S(t)^{-1}$ is bounded as well. Thus, there exists a constant $M$ such that $\left|S(t)^{-1}\right||S(t)| \leq \gamma$. For the periodic parameter matrix system, we give the following condition

Condition $\left(\mathbf{1}^{\prime}\right)$. Assume there exists a constant $\tau>0$ such that for any $t \in \mathbb{R}, x \in \mathbb{R}^{m}, \tilde{f}(t+\tau, x)=$ $\widetilde{f}(t, x), \widetilde{g}(t+\tau, x)=\widetilde{g}(t, x)$. There exist constants $\widetilde{C_{0}}, \widetilde{\beta_{1}}, \widetilde{\beta_{2}}>0$ with $\widetilde{\beta_{1}} \gamma+\frac{{\widetilde{\beta_{2}}}^{2} \gamma^{2}}{2}<\left|\lambda_{1}\right|$, such that for any $s, t \in \mathbb{R}$ and $x, y \in \mathbb{R}^{m}$,

$$
\begin{gathered}
|\widetilde{f}(s, x)-\widetilde{f}(t, y)| \leq \widetilde{C_{0}}|s-t|^{1 / 2}+\widetilde{\beta_{1}}|x-y| \\
|\widetilde{g}(s, x)-\widetilde{g}(t, y)| \leq \widetilde{C_{0}}|s-t|^{1 / 2}+\widetilde{\beta_{2}}|x-y|
\end{gathered}
$$

From this condition it follows that for any $x \in \mathbb{R}^{m}$, the linear growth condition also holds $|\tilde{f}(t, x)| \leq$ $\widetilde{\beta_{1}}|x|+\widetilde{C_{1}},|\widetilde{g}(t, x)| \leq \widetilde{\beta_{2}}|x|+\widetilde{C_{2}}$, where the constants $\widetilde{C_{1}}, \widetilde{C_{2}}>0$, which are independent of time $t$.

Theorem 6.3. Assume that Conditions $\left(A^{\prime}\right),\left(1^{\prime}\right)$. Then there exists a unique random periodic solution $X_{r}^{*} \in L^{2}(\Omega)$ of period $2 \tau$ such that for any initial value $\xi(\omega)$ satisfying Condition (2), the solution of (6.1) satisfies $\lim _{k \rightarrow \infty}\left\|X_{r}^{-2 k \tau}(\xi)-X_{r}^{*}\right\|_{2}=0$. 
Proof. We only need to verify that the corresponding time-invariant system

$$
d Z_{t}^{t_{0}}=B Z_{t}^{t_{0}} d t+f\left(t, Z_{t}^{t_{0}}\right) d t+g\left(t, Z_{t}^{t_{0}}\right) d W_{t},
$$

with $Z_{t_{0}}^{t_{0}}=S\left(t_{0}\right)^{-1} \xi$, where $f(t, x)=S(t)^{-1} \tilde{f}(t, S(t) x), g(t, x)=S(t)^{-1} \widetilde{g}(t, S(t) x)$, satisfies the conditions of Theorem 2.4. It is easy to see that $f(t+2 \tau, x)=f(t, x), g(t+2 \tau, x)=g(t, x)$. For Condition (1), the largest eigenvalue of the matrix $B$ is $\lambda_{1}$. By the Lipschitz condition on function $\widetilde{f}$ and $\widetilde{g}$, we have following result in the time-invariant system $|f(t, x)-f(t, y)| \leq \widetilde{\beta_{1}} \gamma|x-y|$. This means the function $f$ will preserve the Lipschitz property with constant $\beta_{1}=\widetilde{\beta_{1}} \gamma$. Similarly, we can prove that the function $g$ possesses the Lipschitz condition with constant $\beta_{2}=\widetilde{\beta}_{2} \gamma$. Meanwhile, from Condition $\left(1^{\prime}\right)$, we have $\beta_{1}+\frac{\beta_{2}^{2}}{2}<\left|\lambda_{1}\right|$. Moreover, for any $x \in \mathbb{R}^{m}$,

$$
|f(t, x)|=\left|S(t)^{-1} \widetilde{f}(t, S(t) x)\right| \leq \widetilde{\beta_{1}}\left|S(t)^{-1}\right||S(t) x|+\left|S(t)^{-1}\right| \widetilde{C_{1}} \leq \beta_{1}|x|+C_{1} .
$$

Therefore, we can verify the linear growth property of $f$ and $g$ with the constants $C_{1}, C_{2}>0$. The constants $\beta_{1}$ and $\beta_{2}$ are both independent of time $t$. For Condition (2), the initial value of the time-invariant system will preserve the boundedness because of the boundedness of $S(t)^{-1}$. According to Theorem 2.4, there exists a random periodic solution $Z_{r}^{*} \in L^{2}(\Omega)$ with period $2 \tau$ such that $\lim _{k \rightarrow \infty}\left\|Z_{r}^{-2 k \tau}(\xi)-Z_{r}^{*}\right\|_{2}=0$. It turns out that

$$
\lim _{k \rightarrow \infty}\left\|X_{r}^{-2 k \tau}(\xi)-X_{r}^{*}\right\|_{2} \leq\|S(r)\| \lim _{k \rightarrow \infty}\left\|Z_{r}^{-2 k \tau}(\xi)-Z_{r}^{*}\right\|_{2}=0 .
$$

The $2 \tau$-periodicity of $S(r)$ and $Z_{r}^{-2 k \tau}$ give us the random periodicity of solution $X^{*}(r, \omega)$. So $X_{r}^{*}$ is a random periodic solution of (6.1) of period $2 \tau$.

\subsection{Numerical approximation scheme and error estimate}

With the existence of the random periodic solutions, we now consider the scheme to simulate the process $Z$ of equation (6.3). Similar as before, we can consider strong and weak convergence in Euler-Maruyama and modified Milstein methods. Due to the length of the paper, we only consider strong convergence in the Euler scheme given by

$$
\begin{aligned}
\widehat{Z}_{-2 k \tau+(i+1) \Delta t}^{-2 k \tau}= & \widehat{Z}_{-2 k \tau+i \Delta t}^{-2 k \tau}+\left[B \widehat{Z}_{-2 k \tau+i \Delta t}^{-2 k \tau}+S(i \Delta t)^{-1} \tilde{f}\left(i \Delta t, S(i \Delta t) \widehat{Z}_{-2 k \tau+i \Delta t}^{-2 k \tau}\right)\right] \Delta t \\
& +S(i \Delta t)^{-1} \widetilde{g}\left(i \Delta t, S(i \Delta t) \widehat{Z}_{-2 k \tau+i \Delta t}^{-2 k \tau}\right)\left(W_{-2 k \tau+(i+1) \Delta t}-W_{-2 k \tau+i \Delta t}\right) .
\end{aligned}
$$

Theorem 6.4. Assume Conditions $\left(A^{\prime}\right),\left(1^{\prime}\right)$ and (2), $S(t) \in C^{1}(\mathbb{R})$. Then there exists $\widehat{Z}_{r}^{*}$, which is a random periodic solution of period $2 \tau$ for discrete random dynamical system generated from (6.4), such that

$$
\lim _{k \rightarrow \infty}\left\|X_{r}^{-2 k \tau}-S(r) \widehat{Z}_{r}^{-2 k \tau}\right\|_{2} \leq \widetilde{K} \sqrt{\Delta t}, \text { and }\left\|X_{r}^{*}-S(r) \widehat{Z}_{r}^{*}\right\|_{2} \leq \widetilde{K} \sqrt{\Delta t}, r \in[0, T]
$$

for a constant $\widetilde{K}>0$, which is independent of $\Delta t$, where $X_{r}^{*}$ is the exact random periodic solution of (6.1).

Proof. By Theorem 3.4, there exists $\hat{Z}_{r}^{*} \in L^{2}(\Omega)$ such that $\limsup _{k \rightarrow \infty}\left\|\widehat{Z}_{r}^{-2 k \tau}-\widehat{Z}_{r}^{*}\right\|_{2}$

$=0$, where $\widehat{Z}_{r}^{*}$ is the random periodic solution of period $2 \tau$ for discrete random dynamical system generated from (6.4). According to Theorem 4.2, we have the conclusion that there exists a constant 
$K_{1}>0$ such that $\lim _{k \rightarrow \infty}\left\|X_{r}^{-2 k \tau}-S(r) \widehat{Z}_{r}^{-2 k \tau}\right\|_{2} \leq K_{1}\|S(r)\|_{2} \sqrt{\Delta \tilde{t}} \leq \widetilde{K} \sqrt{\Delta t}$. Thus, it follows that

$$
\begin{aligned}
\left\|X_{r}^{*}-S(r) \widehat{Z}_{r}^{*}\right\|_{2} \leq & \limsup _{k \rightarrow \infty}\left\|X_{r}^{*}-X_{r}^{-2 k \tau}\right\|_{2}+\limsup _{k \rightarrow \infty}\left\|X_{r}^{-2 k \tau}-S(r) \widehat{Z}_{r}^{-2 k \tau}\right\|_{2} \\
& +\limsup _{k \rightarrow \infty}\left\|S(r) \widehat{Z}_{r}^{-2 k \tau}-S(r) \widehat{Z}_{r}^{*}\right\|_{2} \leq \widetilde{K} \sqrt{\Delta t} .
\end{aligned}
$$

\section{Acknowledgements}

We sincerely thank the anonymous referees for their constructive comments, which result in significant improvements of the paper. CF and HZ would like to acknowledge the financial support of Royal Society Newton Advanced Fellowship Grant NA150344.

Open Access. This article is distributed under the terms of the Creative Commons Attribution 4.0 International License (http://creativecommons.org/licenses/by/4.0/), which permits unrestricted use, distribution, and reproduction in any medium, provided you give appropriate credit to the original author(s) and the source, provide a link to the Creative Commons license, and indicate if changes were made.

\section{References}

[1] Bao, J., Yuan, C.: Numerical approximation of stationary distributions for stochastic partial differential equations. J. Appl. Probab. 51(3), 858-873 (2014)

[2] Bates, P.W., Lu, K.N., Wang, B.X.: Attractors of non-autonomous stochastic lattice systems in weighted spaces. Physica D 289, 32-50 (2014)

[3] Chekroun, M.D., Simonnet, E., Ghil, M.: Stochastic climate dynamics: random attractors and time-dependent invariant measures. Physica D 240, 1685-1700 (2011)

[4] Cherubini, A.M., Lamb, J.S.W., Rasmussen, M., Sato, Y.: A random dynamical systems perspective on stochastic resonance. Nonlinearity 30, 2835-2853 (2017)

[5] Feng, C., Zhao, H., Zhou, B.: Pathwise random periodic solutions of stochastic differential equations. J. Differ. Equ. 251, 119-149 (2011)

[6] Feng, C., Zhao, H.: Random periodic solutions of SPDEs via integral equations and Wiener-Sobolev compact embedding. J. Funct. Anal. 262, 4377-4422 (2012)

[7] Feng, C., Wu, Y., Zhao, H.: Anticipating random periodic solutions-I. SDEs with multiplicative linear noise, 2015. J. Funct. Anal. 271, 365-417 (2016)

[8] Feng, C., Zhao, H.: Random periodic processes, periodic measures and ergodicity (2015). arXiv:1408.1897

[9] Hartman, P.: Ordinary Differential Equations. Society for Industrial and Applied Mathematics, Philadelphia (2002)

[10] Higham, D., Mao, X., Stuart, A.: Exponential mean-square stability of numerical solutions to stochastic differential equations. LMS J. Comput. Math. 6, 297-313 (2003)

[11] Ikeda, N., Watanabe, S.: Stochastic Differential Equations and Diffusion Processes. North Holland-Kodansha, Tokyo (1981)

[12] Jentzen, A., Kloeden, P.: The numerical approximation of stochastic partial differential equations. Milan J. Math. 77, 205-244 (2009)

[13] Jentzen, A., Kloeden, P.: Taylor expansions of solutions of stochastic partial differential equations with additive noise. Ann. Prob. 38, 532-569 (2010)

[14] Kloeden, P.E., Platen, E.: Numerical Solution of Stochastic Differential Equations. Springer, New York (1991)

[15] Kunita, H.: Stochastic Flows and Stochastic Differential Equations. Cambridge University Press, Cambridge (1990)

[16] Mattingly, J., Stuart, A.M., Higham, D.J.: Ergodicity for SDEs and approximations: locally Lipschitz vector fields and degenerate noise. Stoch. Processes Appl. 101(2), 185-232 (2002)

[17] Milstein, G.N.: Numerical Integrations of Stochastic Differential Equations. Kluwer, Dordrecht (1995)

[18] Poincaré, H.: Memoire sur les courbes definier par une equation differentiate. J. Math. Pures Appl. 7, 375-422 (1881)

[19] Poincaré, H.: Memoire sur les courbes definier par une equation differentiate. J. Math. Pures Appl. 8, 251-296 (1882)

[20] Poincaré, H.: Memoire sur les courbes definier par une equation differentiate. J. Math. Pures Appl. 1, 167-244 (1885)

[21] Poincaré, H.: Memoire sur les courbes definier par une equation differentiate. J. Math. Pures Appl. 2, 151-218 (1886) 
[22] Talay, D.: Second order discretization schemes of stochastic differential systems for the computation of the invariant law. Stoch. Stoch. Rep. 29, 13-36 (1990)

[23] Talay, D., Rubaro, L.: Expansion of the global error for numerical schemes solving stochastic differential equations. Stoch. Anal. Appl. 8, 483-509 (1990)

[24] Tocino, A., Ardanuy, R.: Runge-Kutta methods for numerical solution of stochastic differential equations. J. Comput. Appl. Math. 138, 219-241 (2002)

[25] Wang, B.X.: Existence, stability and bifurcation of random complete and periodic solutions of stochastic parabolic equations. Nonlinear Anal. 103, 9-25 (2014)

[26] Yevik, A., Zhao, H.: Numerical approximations to the stationary solutions of stochastic differential equations. SIAM J. Numer. Anal. 49, 1397-1416 (2011)

[27] Yuan, C., Mao, X.: Stability in distribution of numerical solutions for stochastic differential equations. Stoch. Anal. Appl. 22(5), 1133-1150 (2004)

[28] Zhao, H., Zheng, Z.: Random periodic solutions of random dynamical systems. J. Differ. Equ. 246, 2020-2038 (2009)

Chunrong Feng, Yu Liu and Huaizhong Zhao

Department of Mathematical Sciences

Loughborough University

Loughborough LE11 3TU

UK

e-mail: H.Zhao@lboro.ac.uk

Chunrong Feng

e-mail: C.Feng@lboro.ac.uk

Yu Liu

e-mail: Y.Liu4@lboro.ac.uk

(Received: September 8, 2016; revised: June 13, 2017) 